\title{
Systematic Review \\ Predictors of Mortality in Patients with Advanced Cancer-A Systematic Review and Meta-Analysis
}

\author{
Catherine Owusuaa ${ }^{1, *}$, Simone A. Dijkland ${ }^{2}$, Daan Nieboer ${ }^{2}$, Agnes van der Heide ${ }^{2}$ \\ and Carin C. D. van der Rijt ${ }^{1}$ (D) \\ 1 Department of Medical Oncology, Erasmus MC Cancer Institute, P.O. Box 2040, \\ 3000 CA Rotterdam, The Netherlands; c.vanderrijt@erasmusmc.nl \\ 2 Department of Public Health, Erasmus MC, Erasmus University Medical Center, P.O. Box 2040, \\ 3000 CA Rotterdam, The Netherlands; s.dijkland@erasmusmc.nl (S.A.D.); d.nieboer@erasmusmc.nl (D.N.); \\ a.vanderheide@erasmusmc.nl (A.v.d.H.) \\ * Correspondence: c.owusuaa@erasmusmc.nl; Tel.: +31-62-955-2726
}

Citation: Owusuaa, C.; Dijkland, S.A.; Nieboer, D.; van der Heide, A. van der Rijt, C.C.D. Predictors of Mortality in Patients with Advanced Cancer-A Systematic Review and Meta-Analysis. Cancers 2022, 14, 328. https://doi.org/10.3390/ cancers14020328

Academic Editor: Thomas Licht

Received: 1 December 2021

Accepted: 7 January 2022

Published: 11 January 2022

Publisher's Note: MDPI stays neutral with regard to jurisdictional claims in published maps and institutional affiliations.

Copyright: (C) 2022 by the authors. Licensee MDPI, Basel, Switzerland. This article is an open access article distributed under the terms and conditions of the Creative Commons Attribution (CC BY) license (https:// creativecommons.org/licenses/by/ $4.0 /)$.
Simple Summary: This systematic review and meta-analysis describes the predictors of mortality in patients with advanced cancer. The results indicate that disease stage, lung cancer, ECOG performance status, age, male sex, Charlson comorbidity score, and other multicomponent prognostic models could aid physicians in timely advance care planning. However, combining these predictors in a prognostic model with adequate performance requires more research.

\begin{abstract}
To timely initiate advance care planning in patients with advanced cancer, physicians should identify patients with limited life expectancy. We aimed to identify predictors of mortality. To identify the relevant literature, we searched Embase, MEDLINE, Cochrane Central, Web of Science, and PubMed databases between January 2000-April 2020. Identified studies were assessed on riskof-bias with a modified QUIPS tool. The main outcomes were predictors and prediction models of mortality within a period of 3-24 months. We included predictors that were studied in $\geq 2$ cancer types in a meta-analysis using a fixed or random-effects model and summarized the discriminative ability of models. We included 68 studies (ranging from 42 to 66,112 patients), of which 24 were low risk-of-bias, and 39 were included in the meta-analysis. Using a fixed-effects model, the predictors of mortality were: the surprise question, performance status, cognitive impairment, (sub)cutaneous metastases, body mass index, comorbidity, serum albumin, and hemoglobin. Using a randomeffects model, predictors were: disease stage IV (hazard ratio [HR] 7.58; 95\% confidence interval [CI] 4.00-14.36), lung cancer (HR 2.51; 95\% CI 1.24-5.06), ECOG performance status 1+ (HR 2.03; 95\% CI 1.44-2.86) and 2+ (HR 4.06; 95\% CI 2.36-6.98), age (HR 1.20; 95\% CI 1.05-1.38), male sex (HR 1.24; 95\% CI 1.14-1.36), and Charlson comorbidity score 3+ (HR 1.60; 95\% CI 1.11-2.32). Thirteen studies reported on prediction models consisting of different sets of predictors with mostly moderate discriminative ability. To conclude, we identified reasonably accurate non-tumor specific predictors of mortality. Those predictors could guide in developing a more accurate prediction model and in selecting patients for advance care planning.
\end{abstract}

Keywords: advance care planning; predictors; mortality; advanced cancer

\section{Introduction}

Advance care planning is important in patients with cancer because it enables physicians and patients to discuss and make plans for future health care [1]. It should be initiated timely in patients with advanced cancer, as a decline in the functional status of the patient, although anticipated, often occurs quickly [2]. Therefore, it is a prerequisite for the responsible physician to have insight into the limited life expectancy of their patients.

It is often suggested that patients who are likely to have a maximum life expectancy of 12 months are candidates for advance care planning. Therefore, a period of 12 months 
before death may be typical for patients with palliative care needs and has been included as such in quality standards worldwide [3,4]. The identification of such patients can be based on the surprise question, 'Would you be surprised if this patient died within the next 12 months?' [5]. Although the surprise question was initially developed to encourage physicians to refer their patients for an advance care planning program, it has also been studied as a prediction model for 12-month mortality [6]. However, it has an accuracy ranging from poor to reasonable, with a pooled accuracy level of $78.6 \%$ (95\% confidence interval 69.7-86.3) for patients with cancer [7]. Other prediction models such as the Palliative Prognostic Index, the Palliative Prognostic Score, and the Palliative Performance Scale focus on a life expectancy of only weeks or a few months, which is probably too short for adequate advance care planning procedures [8].

Better insight into whether patients are likely to die within a year might aid physicians in the timely initiation of advance care planning in all patients with advanced cancer who qualify for and would benefit from it. We aimed to provide an overview of predictors and prediction models for mortality within a period of 3-24 months for patients with advanced cancer. By examining all cancer types, we intended to identify general predictors that can be applied by various caregivers.

\section{Materials and Methods}

\subsection{Search Strategy and Selection Criteria}

The search strategy, which included the terms "advanced cancer", "mortality", "death", "predictor", and "prediction model", was developed by one researcher (CO) and an information specialist at the Erasmus MC Medical Library. They performed the search in April 2020 in the following online databases: Embase, MEDLINE, Cochrane Central, Web of Science, and PubMed (Table S1). An online study protocol for this systematic review and meta-analysis (including a search for chronic pulmonary diseases) was published at PROSPERO with registration number CRD42016038494; link: https: / / www.crd.york.ac.uk/PROSPERO/display_record.php?RecordID=38494 (accessed on 6 January 2022).

Studies included in this systematic review and meta-analysis had a population with (predominantly) locally advanced or metastatic cancer; studied mortality within a period of 3-24 months or found a survival between 3-24 months; reported on risk estimates (hazard ratio, odds ratio or relative risk) with their corresponding standard errors or reported on the performance of a prediction model (discriminative ability: area under the receiver operating characteristic curve [AUC], c-statistic, sensitivity, or specificity); and were published in English and from the year 2000 onwards. Both cohort studies and randomized controlled trials were assessed. We excluded systematic reviews and studies that examined mortality or survival outside the period of 3-24 months; only included patients who were treated with curative intent; had a combined outcome (e.g., mortality or hospitalization); or did not report standard errors.

The titles of papers that resulted from the search were downloaded into a reference management software program (EndNote, version X9) and screened by C.O. [9]. Afterwards, C.O. and S.A.D. independently reviewed abstracts and full-text articles to determine the studies' inclusion. If they disagreed, the full article was reassessed, and if necessary, two other researchers (A.v.d.H. and C.C.D.v.d.R.) were involved. When duplicate study data were found in different papers, only the most recent publication was included. We requested full articles that were unavailable to us from the first author through email. We also performed a grey literature search using the bibliographies from included studies and from systematic reviews for additional relevant studies.

\subsection{Data Extraction}

C.O. and S.A.D. independently extracted the following data from each included study: first author, publication year, study design, study population (number of patients, age, sex, and cancer type), follow-up period, mortality rate, overall survival, risk estimates 
and standard errors, and the predictors and discriminative ability of a prediction model. Means were derived with standard deviations and medians with (interquartile) range. When studies reported on different follow-up periods, we only extracted the data of the longest follow-up period within the inclusion criteria. All extracted data were entered in a specifically designed Microsoft Access database.

C.O. and S.A.D. also independently extracted data to assess the risk-of-bias of individual studies using the Quality in Prognosis Studies with adapted items from the Critical Appraisal and Data Extraction for Systematic Reviews of Prediction Modelling Studies checklist $[10,11]$. A total of 23 items were divided over the following six domains: study participation, study attrition, predictor measurement, outcome measurement, statistical analysis and confounding, and model performance (Table S2). All items were given 2 points if bias was not present, 1 point if bias was possibly present, and 0 points if bias was obviously present. The overall risk-of-bias (low [ $\geq 80 \%$ of total points], moderate [ $\geq 60-79 \%$ of total points], or high [ $<60 \%$ of total points]) of each study was generated from the points given to the items per domain. Disagreements in scoring were resolved by discussion between the researchers. This study was conducted according to the Preferred Reporting Items for Systematic and Meta-analyses (PRISMA) guideline [12].

\subsection{Data Analysis}

The main outcomes were predictors and prediction models for mortality within a period of 3-24 months in patients with advanced cancer. The risk estimates and standard errors of predictors that were retrieved from multivariable analyses in published studies were included in the meta-analysis, provided that they were studied in $\geq 2$ (various) cancer types [13]. We calculated the pooled overall risk estimate and standard error with a fixed-effects model for predictors that were pooled from two studies and with a random-effects model for predictors pooled from three or more studies. The fixed-effects model was preferred over the random-effects model if two studies were available, as estimates of heterogeneity become unreliable in a meta-analysis with a few studies. When applying a random-effects model, we calculated the between-study heterogeneity with the $\mathrm{I}^{2}$-statistic [14]. A heterogeneity of $0-30 \%$ was interpreted as insignificant, $40-60 \%$ as moderate, and $>60 \%$ as substantial. We also performed a meta-analysis with only low risk-of-bias studies applying a fixed or random-effects model where appropriate. For performance status, we mapped different cut-offs of the Karnofsky performance scale with the Eastern Cooperative Oncology Group (ECOG) performance status [15]. All metaanalyses were conducted with the Review Manager 5.3 software (Cochrane Collaboration's Information Management System).

For the prediction models that were studied in $\geq 2$ (various) cancer types, we summarized the predictors and model performances. The discriminative ability measured with the AUC or c-statistic ranges from 0.5 to 1, and the closer the AUC or c-statistic is to 1 , the better a model can discriminate patients who are likely to die from those who are not $[16,17]$. Furthermore, we identified publication bias using funnel plots [18].

\section{Results}

The literature search identified 6436 unique studies, of which 6265 were excluded based on their title and abstract (Figure 1). A total of 171 studies were screened for full text. Eventually, a total of 68 studies were systematically interpreted and summarized in the qualitative synthesis, whereupon 39 studies were included in the meta-analysis. 


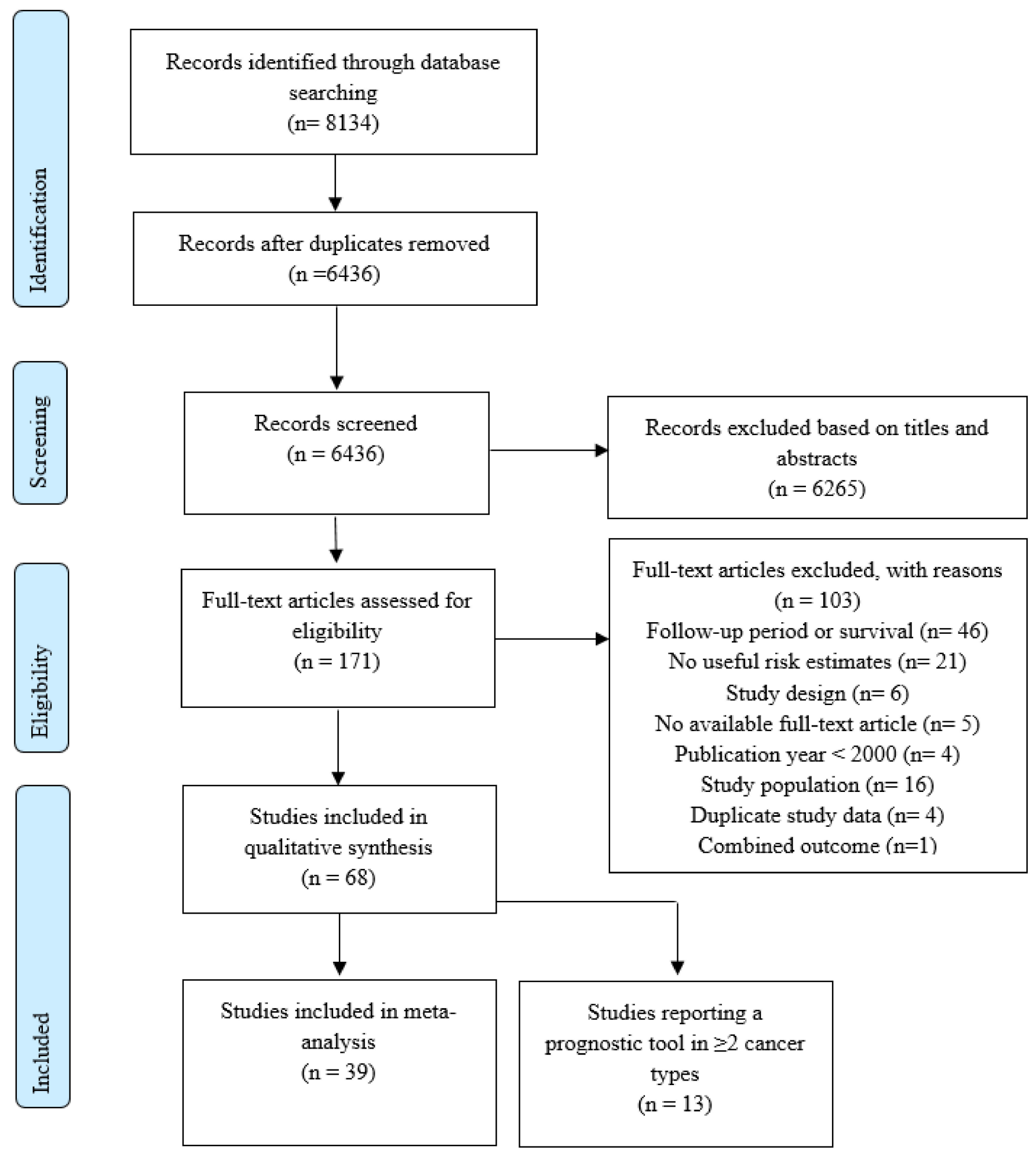

Figure 1. Study selection.

Of the included studies, 63 were cohort studies, and five were analyses from randomized controlled trials. The number of patients ranged from 42 to 66,112 among all studies and from 42 to 13,190 among studies included in the meta-analysis (Table 1). Mean and median reported ages in all studies ranged from 54.0 and 79.4 years. Thirty-three studies had a study population with $\geq 2$ cancer types, whilst the remaining studies included patients with one type of cancer. Mortality rates within the included studies ranged from 8.3 to $94.6 \%$. Twenty-four studies were assessed as low risk-of-bias studies, 38 as moderate risk-of-bias, 
and six as high risk-of-bias (Table 2). Of the risk-of-bias domains, study attrition, which included items regarding loss to follow-up and handling of missing data, was often scored as high risk-of-bias.

In the fixed-effects model, all identified predictors of mortality were significantly associated with mortality (Figure 2). Those predictors were: the surprise question (HR 7.57; 95\% CI 4.41-12.99), ECOG performance status 3-4 (reference [ref]: 0; HR 3.61; 95\% CI 2.58-5.05), presence of (sub)cutaneous metastases (HR 2.10; 95\% CI 1.51-2.93), and the Charlson co-morbidity index score 1-2 (HR 1.28; 95\% CI 1.20-1.37). Body mass index, cognitive impairment (mini-mental state examination score), serum albumin, and hemoglobin were also significant predictors of mortality.

In the random-effects model, we included 14 predictors of mortality, of which 11 were significant (Figure 3 and Figure S1). Due to the low number of studies reporting on categorical variables, we performed a separate meta-analysis for each of the sub-categories. Significant predictors were: disease stage III (pooled from three studies; HR 2.89; 95\% CI 1.42-5.90); disease stage IV (pooled from three studies; HR 7.58; 95\% CI 4.00-14.36); lung cancer (pooled from four studies; HR 2.51; 95\% CI 1.24-5.06). Age per 10-year increase, male sex, and a Charlson comorbidity index score of 3+ were also significant predictors, as were the Karnofsky performance status, ECOG performance status 1+ (ref: 0-1), 1-2 (ref: 0), 2 (ref: 0), and 2+ (ref: 0). Non-significant predictors were ECOG performance status 1 (ref: 0), ECOG performance status (ref: 0-1), and the presence of liver metastases. There was substantial between-study heterogeneity, i.e., $\mathrm{I}^{2}>60 \%$, for the age, ECOG performance status, comorbidity, lung cancer, and liver metastasis. Furthermore, no evidence of major publication bias was identified for each predictor (Figure S2).

In a separate analysis, we only included the predictors found in low risk-of-bias studies, namely age, male sex, and ECOG performance status $1+$, of which only age and male sex were significantly associated with mortality (Table S3). Variables that were excluded for the meta-analyses due to different cut-offs or definitions, or were studied in only one cancer type, are presented in Table S4.

Thirteen studies reported on and analyzed the discriminative ability of 15 different prediction models, which consisted of one variable or combined different clinical variables such as cancer type, performance status, or metastases (Table 3). Most models had a moderate discriminative ability with a c-statistic or AUC ranging from 0.6-0.8, and others, such as the (Oncological-) multidimensional prognostic index, had a good discriminative ability of $>0.8$. The prediction models contained different sets of variables and were examined in just one study, except the surprise question, which was examined in two studies. The external validation of only two models was described.

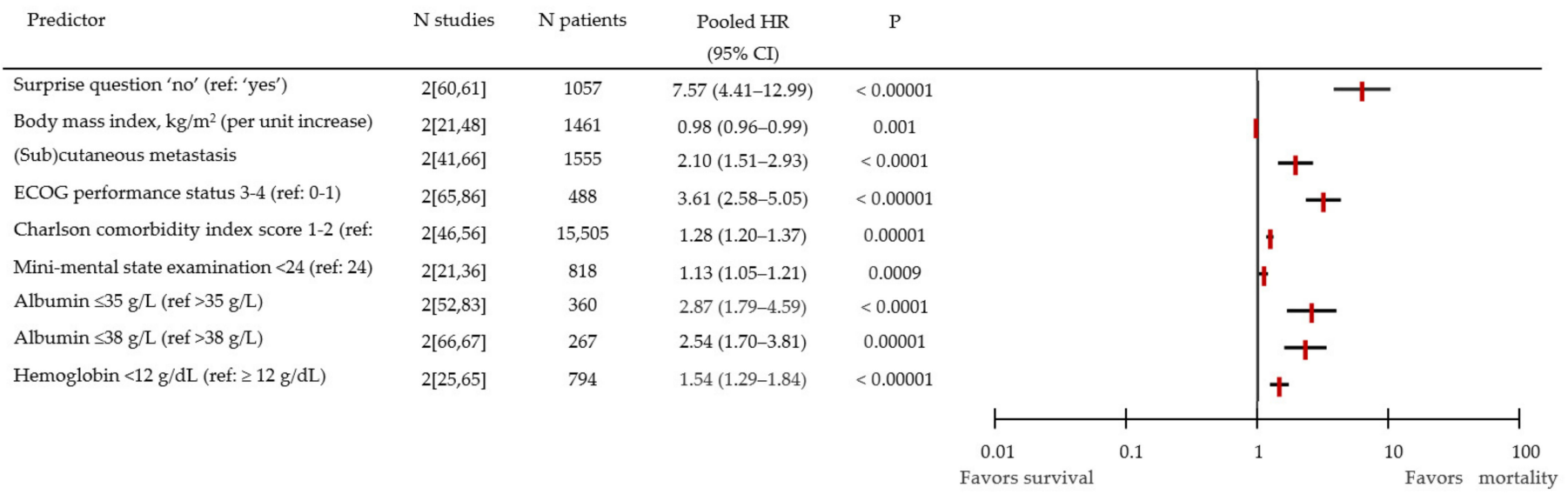

Figure 2. Forest plot of pooled hazard ratios for mortality with a fixed-effects model. CI: confidence interval; HR: hazard ratio; ECOG: Eastern Cooperative Oncology Group. 
Table 1. Study characteristics of all 68 studies reporting predictors of mortality or prediction models.

\begin{tabular}{|c|c|c|c|c|c|c|c|c|c|c|c|}
\hline & $\begin{array}{c}\text { Study } \\
\text { (First Author, } \\
\text { Publication Year) }\end{array}$ & $\begin{array}{l}\text { Study } \\
\text { Design }\end{array}$ & $\begin{array}{c}\mathbf{N} \\
\text { Patients }\end{array}$ & Study Population & Age (Years) & Men (\%) & $\begin{array}{l}\text { Follow-Up } \\
\text { (Months) }\end{array}$ & $\begin{array}{l}\text { Mortality } \\
\text { Rate (\%) }\end{array}$ & $\begin{array}{l}\text { Survival } \\
\text { (Months) }\end{array}$ & $\begin{array}{l}\text { Inclusion in } \\
\text { Meta- } \\
\text { Analysis }\end{array}$ & $\begin{array}{l}\text { Inclusion of } \\
\text { a Model }\end{array}$ \\
\hline 1 & Bartels, 2007 [19] & Cohort & 219 & $\begin{array}{l}\text { Spinal epidural metastases; } \\
\text { various cancer types }\end{array}$ & $62.7 \pm 12.5$ & 58.4 & 10 & $\mathrm{n} / \mathrm{a}$ & $\begin{array}{c}3.0 \\
(0.0-74.4) \\
\end{array}$ & + & + \\
\hline 2 & Braun, $2011[20]$ & Cohort & 1194 & Stage I-IV NSCLC & $\begin{array}{c}58.5 \\
(21.6-86.4)\end{array}$ & 50.3 & $\mathrm{n} / \mathrm{a}$ & 65.2 & $\begin{array}{c}8.8 \\
(8.0-9.5)\end{array}$ & + & - \\
\hline 3 & Brunello, 2016 [21] & Cohort & 658 & Stage I-IV; various cancer types & $77.2 \pm 5.1$ & 34.2 & 12 & 17.4 & $\mathrm{n} / \mathrm{a}$ & + & + \\
\hline 4 & Cesari, 2013 [22] & Cohort & 200 & Stage I-IV ovarian or uterine cancer & $73.5 \pm 6.2$ & 0.0 & 12 & 11.5 & $\mathrm{n} / \mathrm{a}$ & + & + \\
\hline 5 & Chen, 2019 [23] & Cohort & 121 & $\begin{array}{l}\text { Gastric adenocarcinoma; } \\
\text { stage I-IV }\end{array}$ & $64.0 \pm 14.9$ & 60.3 & 12 & 32.2 & $\mathrm{n} / \mathrm{a}$ & - & - \\
\hline 6 & Chow, 2008 [24] & Cohort & 395 & $\begin{array}{c}\text { Metastatic disease, referred for } \\
\text { palliative radiotherapy; various cancer } \\
\text { types }\end{array}$ & $\begin{array}{c}68.0 \\
(31.0-93.0)\end{array}$ & 50.0 & 12 & $\mathrm{n} / \mathrm{a}$ & $\begin{array}{c}4.4 \\
(3.9-6.4)\end{array}$ & + & + \\
\hline 7 & Collette, 2004 [25] & $\mathrm{RCT}$ & 391 & $\begin{array}{l}\text { Metastatic hormone-refractory prostate } \\
\text { cancer }\end{array}$ & $\begin{array}{c}70.8 \\
(34.3-89.3)\end{array}$ & 100.0 & 12 & 42.7 & $\begin{array}{c}10.4 \\
(9.2-11.5)\end{array}$ & + & + \\
\hline 8 & Collins, 2014 [26] & Cohort & 1160 & Malignant glioma & $\mathrm{n} / \mathrm{a}$ & 58.4 & 4 & 23.0 & $\mathrm{n} / \mathrm{a}$ & - & - \\
\hline 9 & $\begin{array}{c}\text { Contreras-Bolívar, } 2019 \\
\text { [27] }\end{array}$ & Cohort & 282 & Various cancer types & $60.4 \pm 12.6$ & 55.7 & 6 & 47.9 & $\mathrm{n} / \mathrm{a}$ & - & - \\
\hline 10 & Deans, 2007 [28] & Cohort & 220 & Stage I-IV gastric or esophageal cancer & $\begin{array}{c}71.0 \\
(62.0-78.0) \\
\end{array}$ & 65.9 & 24 & 61.8 & $13.0(\mathrm{n} / \mathrm{a})$ & + & + \\
\hline 11 & $\begin{array}{l}\text { Dharma-Wardene, } 2004 \\
\text { [29] }\end{array}$ & Cohort & 42 & Stage IIIA/B or IV NSCLC or SCLC & $\begin{array}{c}59.4 \\
(39.0-78.0) \\
\end{array}$ & 45.2 & 24 & 16.7 & $9.9(\mathrm{n} / \mathrm{a})$ & + & - \\
\hline 12 & Efficace, 2006 [30] & RCT & 391 & Stage IIIB-IV NSCLC & $\begin{array}{c}57.0 \\
(28.1-75.9)\end{array}$ & 65.2 & $\mathrm{n} / \mathrm{a}$ & 77.2 & $(6.7-8.9)$ & + & - \\
\hline 13 & Ferrigno, 2001 [31] & Cohort & 343 & $\begin{array}{l}\text { Stage 0-IV bronchogenic } \\
\text { carcinoma }\end{array}$ & $\begin{array}{c}68.0 \\
(39.0-86.0) \\
\end{array}$ & 88.6 & $\begin{array}{c}8.5 \\
(3.6-17.4) \\
\end{array}$ & 79.0 & $\mathrm{n} / \mathrm{a}$ & - & - \\
\hline 14 & Fielding, 2007 [32] & Cohort & 358 & Lung (analysis, stage I-IV) & $\begin{array}{l}\mathrm{n} / \mathrm{a} \text {, subgroups of } \\
\text { different ages } \\
\text { available }\end{array}$ & 75.7 & $\begin{array}{c}3.2 \\
(2.0-7.0)\end{array}$ & 78.5 & $\mathrm{n} / \mathrm{a}$ & + & + \\
\hline 15 & Filippini, 2008 [33] & Cohort & 676 & Newly-diagnosed glioblastoma & $\begin{array}{c}58.0 \\
(16.0-81.0) \\
\end{array}$ & 61.8 & 24 & 84.0 & $\begin{array}{c}13.6 \\
(12.9-14.3) \\
\end{array}$ & + & - \\
\hline 16 & Gagnon, 2013 [34] & Cohort & 258 & Inoperable stage III-IV NSCLC & $\mathrm{n} / \mathrm{a}$ & 50.0 & 12 & $\mathrm{n} / \mathrm{a}$ & $(2.5-18.2)$ & - & + \\
\hline 17 & Geraci, 2006 [35] & Cohort & 372 & $\begin{array}{l}\text { Acutely symptomatic cancer patients; } \\
\text { various cancer types }\end{array}$ & $\begin{array}{c}56.0 \\
(15.0-96.0)\end{array}$ & 49.0 & 6 & 30.0 & $\mathrm{n} / \mathrm{a}$ & - & - \\
\hline
\end{tabular}


Table 1. Cont.

\begin{tabular}{|c|c|c|c|c|c|c|c|c|c|c|c|}
\hline & $\begin{array}{c}\text { Study } \\
\text { (First Author, } \\
\text { Publication Year) }\end{array}$ & $\begin{array}{l}\text { Study } \\
\text { Design }\end{array}$ & $\begin{array}{c}\mathbf{N} \\
\text { Patients }\end{array}$ & Study Population & Age (Years) & Men (\%) & $\begin{array}{l}\text { Follow-Up } \\
\text { (Months) }\end{array}$ & $\begin{array}{l}\text { Mortality } \\
\text { Rate (\%) }\end{array}$ & $\begin{array}{l}\text { Survival } \\
\text { (Months) }\end{array}$ & $\begin{array}{l}\text { Inclusion in } \\
\text { Meta- } \\
\text { Analysis }\end{array}$ & $\begin{array}{l}\text { Inclusion of } \\
\text { a Model }\end{array}$ \\
\hline 18 & Giantin, 2013 [36] & Cohort & 160 & $\begin{array}{l}\text { Inoperable, locally advanced or } \\
\text { metastatic cancer; various } \\
\text { cancer types }\end{array}$ & $79.4 \pm 5.7$ & 45.5 & 12 & 46.9 & $\mathrm{n} / \mathrm{a}$ & + & + \\
\hline 19 & Griguolo, 2018 [37] & Cohort & 668 & $\begin{array}{l}\text { Invasive breast cancer, } \\
\text { brain metastases }\end{array}$ & $\begin{array}{c}56.0 \\
(24.0-85.0)\end{array}$ & 0.1 & $\mathrm{n} / \mathrm{a}$ & 94.6 & $\begin{array}{c}8.1 \\
(6.9-9.4)\end{array}$ & + & - \\
\hline 20 & Gripp, 2007 [38] & Cohort & 216 & $\begin{array}{l}\text { Patients examined for palliative } \\
\text { radiation; various cancer types }\end{array}$ & $\begin{array}{c}64.0 \\
(21.0-96.0)\end{array}$ & 51.0 & 6 & 51.4 & $\mathrm{n} / \mathrm{a}$ & - & - \\
\hline 21 & Gupta, 2004 [39] & Cohort & 58 & Stage IV pancreatic cancer & $56.2 \pm 10.7$ & 60.3 & $\mathrm{n} / \mathrm{a}$ & 72.4 & $(4.9-10.2)$ & - & - \\
\hline 22 & Gupta, 2009 [40] & Cohort & 165 & Stage IIIB or IV NSCLC & $\begin{array}{c}56.0 \\
(30.0-78.0)\end{array}$ & 56.4 & $\mathrm{n} / \mathrm{a}$ & 67.3 & $(6.8-16.8)$ & - & - \\
\hline 23 & Hoang, 2005 [41] & $\mathrm{RCT}$ & 1436 & Stage IIIB or IV NSCLC & $\mathrm{n} / \mathrm{a}$ & 63.0 & 24 & 89.0 & $8.2(\mathrm{n} / \mathrm{a})$ & + & + \\
\hline 24 & Hong, 2016 [42] & Cohort & 183 & Advanced HCC & Mean 55.8 & 86.3 & 6 & 74.9 & $\mathrm{n} / \mathrm{a}$ & - & + \\
\hline 25 & Hosono, 2005 [43] & Cohort & 165 & $\begin{array}{l}\text { Spinal metastases; various } \\
\text { cancer types }\end{array}$ & $\mathrm{n} / \mathrm{a}$ & $\mathrm{n} / \mathrm{a}$ & $\begin{array}{c}23.4 \\
(0.3-140.0)\end{array}$ & $\mathrm{n} / \mathrm{a}$ & $\mathrm{n} / \mathrm{a}$ & - & - \\
\hline 26 & Hui, 2014 [44] & Cohort & 222 & $\begin{array}{l}\text { Advanced cancer, seen by } \\
\text { palliative care mobile team; various } \\
\text { cancer types }\end{array}$ & $\begin{array}{c}55.0 \\
(22.0-79.0)\end{array}$ & 41.0 & $\begin{array}{c}3.9 \\
(0.9-7.9)\end{array}$ & 64.0 & $\begin{array}{c}3.6 \\
(2.3-4.2)\end{array}$ & + & - \\
\hline 27 & Hui, 2016 [45] & Cohort & 216 & $\begin{array}{l}\text { Advanced cancer, seen by the palliative } \\
\text { care mobile team; various cancer types }\end{array}$ & $\begin{array}{c}54.9 \\
(22.0-79.0)\end{array}$ & 42.0 & $\begin{array}{c}7.9 \\
(6.9-8.6)\end{array}$ & 63.0 & $\begin{array}{c}3.6 \\
(2.3-4.4)\end{array}$ & - & + \\
\hline 28 & Iversen, 2009 [46] & Cohort & 13,190 & Stage I-IV colorectal cancer & $\mathrm{n} / \mathrm{a}$ & 47.0 & 12 & $\mathrm{n} / \mathrm{a}$ & $\mathrm{n} / \mathrm{a}$ & + & - \\
\hline 29 & Jang, 2014 [47] & Cohort & 1655 & $\begin{array}{l}\text { Advanced cancer; various } \\
\text { cancer types }\end{array}$ & $\begin{array}{c}65.0 \\
(21.0-97.0)\end{array}$ & 49.0 & $\mathrm{n} / \mathrm{a}$ & 91.0 & $\begin{array}{c}4.4 \\
(4.0-4.7)\end{array}$ & - & + \\
\hline 30 & Jonna, 2016 [48] & Cohort & 803 & Stage I-IV; various cancer types & $72.5(\mathrm{n} / \mathrm{a})$ & 51.8 & 12 & 77.3 & $4.9(\mathrm{n} / \mathrm{a})$ & + & + \\
\hline 31 & Katagiri, 2005 [49] & Cohort & 350 & Bone metastases; various cancer types & $\begin{array}{c}59.0 \\
(14.0-88.0)\end{array}$ & 56.9 & 24 & 67.0 & $\mathrm{n} / \mathrm{a}$ & + & - \\
\hline 32 & Kilgour, 2013 [50] & Cohort & 203 & $\begin{array}{l}\text { Locally, advanced and } \\
\text { metastatic cancer; various } \\
\text { cancer types }\end{array}$ & $64.3 \pm 12.8$ & 57.1 & $\mathrm{n} / \mathrm{a}$ & 76.6 & $\begin{array}{c}7.3 \\
(5.3-9.3)\end{array}$ & - & - \\
\hline 33 & Kim, 2009 [51] & Cohort & 325 & $\begin{array}{c}\text { UICC stage I-IV } \\
\text { newly-diagnosed HCC }\end{array}$ & $58.8 \pm 9.5$ & 80.9 & 24 & 46.4 & $\begin{array}{c}14.7 \\
(2.0-88.0)\end{array}$ & - & + \\
\hline 34 & $\begin{array}{l}\text { Kinoshita, } \\
2012[52]\end{array}$ & Cohort & 133 & Stage I-IV HCC & $\begin{array}{c}71.0 \\
(43.0-87.0)\end{array}$ & 70.7 & $\begin{array}{c}22.0 \\
(1.0-69.0)\end{array}$ & 13.0-91.4 & $\mathrm{n} / \mathrm{a}$ & + & - \\
\hline
\end{tabular}


Table 1. Cont.

\begin{tabular}{|c|c|c|c|c|c|c|c|c|c|c|c|}
\hline & $\begin{array}{c}\text { Study } \\
\text { (First Author, } \\
\text { Publication Year) }\end{array}$ & $\begin{array}{l}\text { Study } \\
\text { Design }\end{array}$ & $\begin{array}{c}\mathbf{N} \\
\text { Patients }\end{array}$ & Study Population & Age (Years) & Men (\%) & $\begin{array}{l}\text { Follow-Up } \\
\text { (Months) }\end{array}$ & $\begin{array}{l}\text { Mortality } \\
\text { Rate (\%) }\end{array}$ & $\begin{array}{l}\text { Survival } \\
\text { (Months) }\end{array}$ & $\begin{array}{l}\text { Inclusion in } \\
\text { Meta- } \\
\text { Analysis }\end{array}$ & $\begin{array}{l}\text { Inclusion o } \\
\text { a Model }\end{array}$ \\
\hline 35 & $\begin{array}{l}\text { Langendijk, } \\
2000[53]\end{array}$ & Cohort & 198 & Stage I-IIIB NSCLC & $\mathrm{n} / \mathrm{a}$ & 85.0 & $\mathrm{n} / \mathrm{a}$ & $\mathrm{n} / \mathrm{a}$ & $(2.8-13.8)$ & - & - \\
\hline 36 & Liljehult, 2017 [54] & Cohort & 109 & Glioblastoma & $65.0 \pm 9.9$ & 56.9 & 12 & 55.0 & $\mathrm{n} / \mathrm{a}$ & - & - \\
\hline 37 & $\begin{array}{l}\text { Limquiaco, } \\
2009[55]\end{array}$ & Cohort & 471 & Stage I-IV HCC & $58.8 \pm 12.2$ & 85.1 & 6 & 45.0 & $10.1 \pm 10.3$ & - & + \\
\hline 38 & Lund, 2009 [56] & Cohort & 2315 & Renal cancer & $\begin{array}{c}\text { Men: 68.0 } \\
(15.0-96.0) ; \\
\text { women: } 70.0 \\
(18.0-97.0)\end{array}$ & 58.7 & 12 & 36.9 & $\mathrm{n} / \mathrm{a}$ & + & - \\
\hline 39 & Maione, 2005 [57] & $\mathrm{RCT}$ & 566 & Stage IIIB-IV NSCLC & $\begin{array}{c}74.0 \\
(70.0-84.0)\end{array}$ & 82.0 & 12 & 68 & $\begin{array}{c}6.9 \\
(6.4-7.8)\end{array}$ & + & - \\
\hline 40 & Marrero, 2005 [58] & Cohort & 244 & Stage I-IV HCC & $57.0 \pm 10.0$ & 73.0 & 12 & 42.0 & $\begin{array}{c}16.4 \\
(12.9-19.8)\end{array}$ & + & - \\
\hline 41 & Martin, 2010 [59] & Cohort & 1164 & $\begin{array}{l}\text { Metastatic cancer; various } \\
\text { cancer types }\end{array}$ & $66.8 \pm 13.0$ & 49.0 & $\begin{array}{c}3.1 \\
(0.0-38.6)\end{array}$ & 86.4 & $\mathrm{n} / \mathrm{a}$ & + & + \\
\hline 42 & Moroni, 2014 [60] & Cohort & 231 & $\begin{array}{l}\text { Advanced cancer; various } \\
\text { cancer types }\end{array}$ & $70.2 \pm 0.9$ & 50.6 & 12 & 45.0 & $\mathrm{n} / \mathrm{a}$ & + & + \\
\hline 43 & Moss, 2010 [61] & Cohort & 826 & $\begin{array}{l}\text { Stage I-IV breast, lung, and } \\
\text { colon cancer }\end{array}$ & $60.0 \pm 13.0$ & 14.8 & 12 & 8.3 & $\mathrm{n} / \mathrm{a}$ & + & + \\
\hline 44 & Motzer, 2004 [62] & Cohort & 251 & Stage IV renal cell carcinoma & $\begin{array}{c}57.0 \\
(23.0-77.0)\end{array}$ & 67.0 & 24 & 76.0 & $\begin{array}{c}10.2 \\
(8.0-12.0)\end{array}$ & + & - \\
\hline 45 & Norman, 2010 [63] & Cohort & 399 & Stage I-IV; various cancer types & $63.0 \pm 11.8$ & 52.1 & 6 & 25.1 & $\mathrm{n} / \mathrm{a}$ & + & - \\
\hline 46 & Orskov, 2016 [64] & Cohort & 2654 & Stage I-IV ovary cancer & $\mathrm{n} / \mathrm{a}$ & 0.0 & 12 & 16.0 & $\mathrm{n} / \mathrm{a}$ & - & - \\
\hline 47 & Park, 2016 [65] & Cohort & 403 & $\begin{array}{l}\text { Metastatic or recurrent } \\
\text { pancreatic ductal } \\
\text { adenocarcinoma }\end{array}$ & $\begin{array}{c}66.0 \\
(29.0-96.0)\end{array}$ & 49.1 & $\begin{array}{c}7.9 \\
(0.1-70.5)\end{array}$ & $\mathrm{n} / \mathrm{a}$ & $\begin{array}{c}8.2 \\
(7.3-9.1)\end{array}$ & + & + \\
\hline 48 & Penel, 2008 [66] & Cohort & 119 & Bone metastases; various cancer types & $\begin{array}{c}57.0 \\
(29.0-84.0)\end{array}$ & 64.7 & 3 & 34.0 & $\begin{array}{c}3.9 \\
(0.0-94.5)\end{array}$ & + & + \\
\hline 49 & Penel, 2008 [67] & Cohort & 148 & $\begin{array}{l}\text { Patients screened for phase } 1 \text { trial; } \\
\text { various cancer types }\end{array}$ & $\begin{array}{c}54.0 \\
(23.0-79.0)\end{array}$ & $\mathrm{n} / \mathrm{a}$ & 3 & 73.0 & $\begin{array}{c}5.7 \\
(0.0-79.6)\end{array}$ & + & - \\
\hline 50 & Pinato, 2015 [68] & Cohort & 97 & Intermediate-advanced HCC & $\begin{array}{c}64.0 \\
(22.0-82.0)\end{array}$ & 80.0 & $\mathrm{n} / \mathrm{a}$ & 71.0 & $\begin{array}{c}5.7 \\
(1.0-88.0)\end{array}$ & - & + \\
\hline
\end{tabular}


Table 1. Cont.

\begin{tabular}{|c|c|c|c|c|c|c|c|c|c|c|c|}
\hline & $\begin{array}{c}\text { Study } \\
\text { (First Author, } \\
\text { Publication Year) }\end{array}$ & $\begin{array}{l}\text { Study } \\
\text { Design }\end{array}$ & $\begin{array}{c}\mathbf{N} \\
\text { Patients }\end{array}$ & Study Population & Age (Years) & Men (\%) & $\begin{array}{l}\text { Follow-Up } \\
\text { (Months) }\end{array}$ & $\begin{array}{l}\text { Mortality } \\
\text { Rate (\%) }\end{array}$ & $\begin{array}{l}\text { Survival } \\
\text { (Months) }\end{array}$ & $\begin{array}{l}\text { Inclusion in } \\
\text { Meta- } \\
\text { Analysis }\end{array}$ & $\begin{array}{l}\text { Inclusion of } \\
\text { a Model }\end{array}$ \\
\hline 51 & Pointillart, 2011 [69] & Cohort & 142 & $\begin{array}{c}\text { Vertebral metastases; various cancer } \\
\text { types }\end{array}$ & $\begin{array}{c}61.8 \\
(28.0-89.0)\end{array}$ & 57.0 & 12 & 50.7 & $5.0(\mathrm{n} / \mathrm{a})$ & + & - \\
\hline 52 & Roychowdhury, 2003 [70] & Cohort & 364 & $\begin{array}{l}\text { Locally advanced or metastatic } \\
\text { urothelial transitional-cell } \\
\text { carcinoma }\end{array}$ & $63.5(\mathrm{n} / \mathrm{a})$ & 79.1 & $\mathrm{n} / \mathrm{a}$ & $\mathrm{n} / \mathrm{a}$ & $\begin{array}{c}14.2 \\
(13.1-16.8)\end{array}$ & - & - \\
\hline 53 & Rydzek, 2015 [71] & Cohort & 326 & $\begin{array}{c}\text { Breast cancer, SCLC or NSCLC; patients } \\
\text { with established } \\
\text { cardiovascular disease }\end{array}$ & $67.8 \pm 10.0$ & 54.0 & 12 & $\mathrm{n} / \mathrm{a}$ & $(4.8-96.0)$ & + & - \\
\hline 54 & $\begin{array}{l}\text { Schoenfeld, } \\
2020[72]\end{array}$ & Cohort & 1216 & Various cancer types, spinal metastases & $58.0 \pm 9.7$ & 50.0 & 12 & 47.0 & $\begin{array}{c}8.4 \\
(3.1-21.1)\end{array}$ & - & - \\
\hline 55 & Scott, $2002[73]$ & Cohort & 106 & Stage III-IV NSCLC & $\begin{array}{c}69.0 \\
(43.0-87.0) \\
\end{array}$ & 58.5 & $\mathrm{n} / \mathrm{a}$ & $\mathrm{n} / \mathrm{a}$ & $\begin{array}{c}5.2 \\
(0.3-38.5) \\
\end{array}$ & + & - \\
\hline 56 & Seow, 2013 [74] & Cohort & 11,342 & $\begin{array}{c}\text { Various cancer types, patients with PPS } \\
\text { assessment }\end{array}$ & $64.0 \pm \mathrm{n} / \mathrm{a}$ & 45.6 & 6 & 25 & $\mathrm{n} / \mathrm{a}$ & + & - \\
\hline 57 & Shen, 2007 [75] & Cohort & 49 & HCC, patients underwent TACE & $57.0 \pm 1.0$ & 81.6 & $\mathrm{n} / \mathrm{a}$ & 49.0 & $\begin{array}{c}12.0 \\
(1.0-72.0)\end{array}$ & - & - \\
\hline 59 & $\begin{array}{l}\text { Sutradhar, } \\
2014 \text { [77] }\end{array}$ & Cohort & 66,112 & Various cancer types & $\mathrm{n} / \mathrm{a}$ & 43.8 & $19.3(\mathrm{n} / \mathrm{a})$ & 26.3 & $\mathrm{n} / \mathrm{a}$ & - & + \\
\hline 60 & Suzuki, $2020[78]$ & Cohort & 185 & Advanced urothelial cancer & $\begin{array}{c}70.0 \\
(64.0-76.0)\end{array}$ & 68.0 & 12 & 71.9 & 14.9 (n/a) & - & - \\
\hline 61 & Tsai, 2014 [79] & Cohort & 522 & $\begin{array}{l}\text { Advanced cancer; various } \\
\text { cancer types }\end{array}$ & $60.6 \pm 13.2$ & 61.7 & 6 & 91.8 & $\mathrm{n} / \mathrm{a}$ & - & - \\
\hline 62 & $\begin{array}{l}\text { Tripodoro, } \\
2019[80]\end{array}$ & Cohort & 317 & Various cancer types, stage III or IV & $\begin{array}{c}77.0 \\
(21.0-99.0)\end{array}$ & 33.4 & 24 & 74.8 & $4.0(\mathrm{n} / \mathrm{a})$ & - & - \\
\hline 63 & Ueno, 2000 [81] & Cohort & 103 & Metastatic pancreatic cancer & $\begin{array}{c}62.0 \\
(42.0-75.0)\end{array}$ & 68.0 & 12 & 94.4 & $3.2(\mathrm{n} / \mathrm{a})$ & + & - \\
\hline 64 & van der Linden, 2005 [82] & $\mathrm{RCT}$ & 342 & $\begin{array}{l}\text { Spinal metastases; various } \\
\text { cancer types }\end{array}$ & $\begin{array}{c}66.0 \\
(34.0-90.0)\end{array}$ & 53.0 & 24 & 75.0 & $\begin{array}{c}11.0 \\
(10.0-12.0)\end{array}$ & - & - \\
\hline 65 & Vigano, 2000 [83] & Cohort & 227 & $\begin{array}{c}\text { Inoperable, recurrent, } \\
\text { progressive or metastatic } \\
\text { cancer; various cancer types }\end{array}$ & $\begin{array}{c}62.0 \\
(29.0-92.0)\end{array}$ & 36.1 & 20 & 91.6 & $5.8(\mathrm{n} / \mathrm{a})$ & + & - \\
\hline
\end{tabular}


Table 1. Cont.

\begin{tabular}{|c|c|c|c|c|c|c|c|c|c|c|c|}
\hline & $\begin{array}{c}\text { Study } \\
\text { (First Author, } \\
\text { Publication Year) }\end{array}$ & $\begin{array}{l}\text { Study } \\
\text { Design }\end{array}$ & $\begin{array}{c}\mathbf{N} \\
\text { Patients }\end{array}$ & Study Population & Age (Years) & Men (\%) & $\begin{array}{l}\text { Follow-Up } \\
\text { (Months) }\end{array}$ & $\begin{array}{l}\text { Mortality } \\
\text { Rate (\%) }\end{array}$ & $\begin{array}{l}\text { Survival } \\
\text { (Months) }\end{array}$ & $\begin{array}{l}\text { Inclusion in } \\
\text { Meta- } \\
\text { Analysis }\end{array}$ & $\begin{array}{l}\text { Inclusion of } \\
\text { a Model }\end{array}$ \\
\hline 66 & Villa, 2011 [84] & Cohort & 285 & $\begin{array}{l}\text { Newly-diagnosed brain } \\
\text { metastases; various cancer types }\end{array}$ & $\begin{array}{c}62.0 \\
(20.0-90.0)\end{array}$ & $\mathrm{n} / \mathrm{a}$ & 12 & 82.4 & $\mathrm{n} / \mathrm{a}$ & + & + \\
\hline 67 & Wei, 2019 [85] & Cohort & 71 & $\begin{array}{c}\text { Pneumonic-type adenocarcinoma, } 90 \% \\
\text { with stage IIIB or IV }\end{array}$ & $\begin{array}{c}62.0 \\
(25.0-91.0)\end{array}$ & 45.1 & 6 & 84.5 & $\begin{array}{c}7.5 \\
(1.0-42.0)\end{array}$ & + & - \\
\hline 68 & $\begin{array}{l}\text { Yamashita, } \\
2011[86]\end{array}$ & Cohort & 85 & $\begin{array}{l}\text { Spinal metastases; various } \\
\text { cancer types }\end{array}$ & $60.3 \pm 11.6$ & 51.8 & 12 & 51.8 & $\mathrm{n} / \mathrm{a}$ & - & - \\
\hline
\end{tabular}

H: high risk-of-bias; HCC: hepatocellular carcinoma; L: low risk-of-bias; M: moderate risk-of-bias; N: number of patients; n/a: not available; NSCLC: non-small cell lung cancer; PPS: Palliative Performance Scale; RCT: randomized controlled trial; SCLC: small cell lung cancer; TACE: trans arterial chemoembolization; UICC: Union Internationale Contre le Cancer. 
Table 2. Summary of risk-of-bias assessment.

\begin{tabular}{|c|c|c|c|c|c|c|c|}
\hline Study & $\begin{array}{c}\text { Study } \\
\text { Participation }\end{array}$ & Study Attrition & Predictors & Outcome & $\begin{array}{l}\text { Statistical } \\
\text { Analysis and } \\
\text { Cofounding }\end{array}$ & $\begin{array}{l}\text { Performance of } \\
\text { Prediction Tool }\end{array}$ & Overall Bias \\
\hline Bartels, 2007 [19] & $\mathrm{L}$ & $\mathrm{H}$ & M & $\mathrm{L}$ & $\mathrm{L}$ & M & M \\
\hline Braun, 2011 [20] & $\bar{L}$ & $\mathrm{H}$ & $\mathrm{L}$ & $\mathrm{L}$ & $\mathrm{L}$ & $\mathrm{n} / \mathrm{a}$ & $\mathrm{L}$ \\
\hline Brunello, 2016 [21] & $\mathrm{L}$ & $\mathrm{H}$ & $\mathrm{L}$ & $\mathrm{L}$ & $\mathrm{M}$ & M & $\bar{M}$ \\
\hline Cesari, 2013 [22] & $\mathrm{L}$ & $\mathrm{H}$ & $\mathrm{L}$ & $\mathrm{L}$ & M & M & M \\
\hline Chen, 2019 [23] & $\mathrm{L}$ & M & $\mathrm{L}$ & $\mathrm{L}$ & $\mathrm{H}$ & $\mathrm{n} / \mathrm{a}$ & M \\
\hline Chow, 2008 [24] & $\mathrm{L}$ & $\mathrm{H}$ & $\mathrm{L}$ & M & M & $\mathrm{L}$ & M \\
\hline Collette, 2004 [25] & $\bar{M}$ & $\mathrm{H}$ & $\mathrm{L}$ & $\mathrm{L}$ & $\mathrm{L}$ & $\mathrm{M}$ & M \\
\hline Collins, 2014 [26] & $\mathrm{L}$ & M & $\mathrm{L}$ & $\mathrm{L}$ & $\mathrm{L}$ & $\mathrm{n} / \mathrm{a}$ & $\mathrm{L}$ \\
\hline $\begin{array}{c}\text { Contreras-Bolívar, } \\
2019 \text { [27] }\end{array}$ & $\mathrm{L}$ & M & $\mathrm{L}$ & $\mathrm{L}$ & $\mathrm{L}$ & $\mathrm{n} / \mathrm{a}$ & $\mathrm{L}$ \\
\hline Deans, 2007 [28] & $\mathrm{L}$ & M & $\mathrm{L}$ & $\mathrm{L}$ & $\mathrm{L}$ & M & $\mathrm{L}$ \\
\hline $\begin{array}{c}\text { Dharma-Wardene, } \\
2004 \text { [29] }\end{array}$ & $\mathrm{L}$ & $\mathrm{H}$ & $\mathrm{L}$ & $\mathrm{L}$ & M & $\mathrm{n} / \mathrm{a}$ & $\mathrm{M}$ \\
\hline Efficace, 2006 [30] & $\mathrm{L}$ & $\mathrm{H}$ & M & $\mathrm{L}$ & $\mathrm{L}$ & $\mathrm{n} / \mathrm{a}$ & M \\
\hline Ferrigno, 2001 [31] & $\mathrm{L}$ & $\mathrm{H}$ & $\mathrm{L}$ & $\mathrm{L}$ & $\mathrm{L}$ & $\mathrm{n} / \mathrm{a}$ & $\mathrm{L}$ \\
\hline Fielding, 2007 [32] & $\mathrm{L}$ & $\mathrm{H}$ & $\mathrm{L}$ & $\mathrm{L}$ & $\mathrm{L}$ & $\mathrm{M}$ & M \\
\hline Filippini, 2008 [33] & $\mathrm{L}$ & M & M & $\mathrm{L}$ & $\mathrm{L}$ & $\mathrm{n} / \mathrm{a}$ & $\mathrm{L}$ \\
\hline Gagnon, 2013 [34] & $\mathrm{L}$ & $\mathrm{H}$ & $\mathrm{L}$ & M & $\mathrm{L}$ & $\mathrm{L}$ & $\mathrm{M}$ \\
\hline Geraci, 2006 [35] & $\mathrm{L}$ & $\mathrm{H}$ & $\mathrm{L}$ & $\mathrm{L}$ & $\mathrm{L}$ & $\mathrm{n} / \mathrm{a}$ & $\mathrm{L}$ \\
\hline Giantin, 2013 [36] & $\mathrm{L}$ & $\mathrm{H}$ & $\mathrm{L}$ & $\mathrm{L}$ & $\mathrm{L}$ & $\mathrm{M}$ & M \\
\hline Griguolo, 2018 [37] & $\mathrm{L}$ & $\mathrm{H}$ & $\mathrm{L}$ & $\mathrm{L}$ & $\mathrm{L}$ & $\mathrm{n} / \mathrm{a}$ & $\mathrm{L}$ \\
\hline Gripp, 2007 [38] & $\mathrm{L}$ & $\mathrm{H}$ & $\mathrm{L}$ & $\mathrm{L}$ & $\mathrm{L}$ & $\mathrm{n} / \mathrm{a}$ & $\mathrm{L}$ \\
\hline Gupta, 2004 [39] & $\mathrm{L}$ & $\mathrm{H}$ & $\mathrm{L}$ & $\mathrm{L}$ & $\mathrm{L}$ & $\mathrm{n} / \mathrm{a}$ & $\mathrm{L}$ \\
\hline Gupta, 2009 [40] & $\bar{L}$ & M & $\mathrm{L}$ & $\bar{L}$ & $\mathrm{M}$ & $\mathrm{n} / \mathrm{a}$ & $\mathrm{L}$ \\
\hline Hoang, 2005 [41] & $\mathrm{L}$ & $\mathrm{H}$ & M & $\mathrm{L}$ & $\mathrm{L}$ & $\mathrm{H}$ & $\mathrm{H}$ \\
\hline Hong, 2016 [42] & $\mathrm{L}$ & $\mathrm{H}$ & $\mathrm{L}$ & $\mathrm{L}$ & $\mathrm{M}$ & M & M \\
\hline Hosono, 2005 [43] & $\bar{M}$ & $\mathrm{H}$ & $\mathrm{M}$ & $\bar{L}$ & M & $\mathrm{n} / \mathrm{a}$ & $\mathrm{H}$ \\
\hline Hui, 2014 [44] & $\mathrm{L}$ & $\mathrm{H}$ & $\mathrm{L}$ & $\mathrm{L}$ & $\mathrm{L}$ & $\mathrm{n} / \mathrm{a}$ & $\mathrm{L}$ \\
\hline Hui, 2016 [45] & $\mathrm{L}$ & $\mathrm{H}$ & $\mathrm{L}$ & $\mathrm{L}$ & $\mathrm{H}$ & $\mathrm{M}$ & $\mathrm{H}$ \\
\hline Iversen, 2009 [46] & $\mathrm{L}$ & $\mathrm{H}$ & $\mathrm{L}$ & $\mathrm{L}$ & M & $\mathrm{n} / \mathrm{a}$ & M \\
\hline Jang, 2014 [47] & $\mathrm{L}$ & $\mathrm{H}$ & $\mathrm{L}$ & $\mathrm{L}$ & $\mathrm{H}$ & $\mathrm{M}$ & $\mathrm{H}$ \\
\hline Jonna, 2016 [48] & $\mathrm{L}$ & $\mathrm{H}$ & $\mathrm{M}$ & $\mathrm{L}$ & $\mathrm{L}$ & M & M \\
\hline Katagiri, 2005 [49] & $\mathrm{L}$ & $\mathrm{H}$ & M & $\mathrm{L}$ & M & $\mathrm{n} / \mathrm{a}$ & M \\
\hline Kilgour, 2013 [50] & $\mathrm{L}$ & $\mathrm{L}$ & $\mathrm{L}$ & $\mathrm{L}$ & M & $\mathrm{n} / \mathrm{a}$ & $\mathrm{L}$ \\
\hline Kim, 2009 [51] & $\mathrm{L}$ & $\mathrm{H}$ & M & $\mathrm{L}$ & M & $\mathrm{M}$ & $\mathrm{H}$ \\
\hline Kinoshita, 2012 [52] & $\mathrm{L}$ & $\mathrm{H}$ & $\mathrm{L}$ & $\mathrm{L}$ & M & $\mathrm{n} / \mathrm{a}$ & M \\
\hline Langendijk, 2000 [53] & $\bar{L}$ & $\mathrm{H}$ & $\mathrm{L}$ & M & M & $\mathrm{n} / \mathrm{a}$ & M \\
\hline Liljehult, 2017 [54] & M & $\mathrm{H}$ & $\mathrm{L}$ & $\mathrm{L}$ & M & $\mathrm{n} / \mathrm{a}$ & M \\
\hline Limquiaco, 2009 [55] & $\mathrm{L}$ & $\mathrm{H}$ & $\mathrm{L}$ & $\mathrm{L}$ & $\mathrm{L}$ & $\mathrm{M}$ & M \\
\hline Lund, 2009 [56] & $\mathrm{L}$ & M & $\mathrm{L}$ & $\mathrm{L}$ & $\overline{\mathrm{H}}$ & $\mathrm{n} / \mathrm{a}$ & M \\
\hline Maione, 2005 [57] & $\mathrm{L}$ & $\mathrm{H}$ & $\mathrm{L}$ & $\mathrm{L}$ & M & $\mathrm{n} / \mathrm{a}$ & M \\
\hline Marrero, 2005 [58] & $\mathrm{L}$ & M & M & $\mathrm{L}$ & M & $\mathrm{n} / \mathrm{a}$ & M \\
\hline Martin, 2010 [59] & $\mathrm{L}$ & $\mathrm{H}$ & $\mathrm{L}$ & $\mathrm{L}$ & $\mathrm{L}$ & $\mathrm{L}$ & $\mathrm{L}$ \\
\hline Moroni, 2014 [60] & $\mathrm{L}$ & $\mathrm{H}$ & $\mathrm{L}$ & $\mathrm{L}$ & $\mathrm{L}$ & M & M \\
\hline Moss, 2010 [61] & $\mathrm{L}$ & $\mathrm{H}$ & $\mathrm{L}$ & $\mathrm{L}$ & $\mathrm{L}$ & M & M \\
\hline Motzer, 2004 [62] & $\bar{L}$ & $\mathrm{H}$ & $\mathrm{L}$ & $\bar{L}$ & $\bar{L}$ & $\mathrm{n} / \mathrm{a}$ & $\mathrm{L}$ \\
\hline Norman, 2010 [63] & $\mathrm{L}$ & $\mathrm{H}$ & $\mathrm{L}$ & $\mathrm{L}$ & M & $\mathrm{n} / \mathrm{a}$ & M \\
\hline Orskov, 2016 [64] & $\mathrm{L}$ & $\mathrm{M}$ & $\mathrm{L}$ & $\mathrm{L}$ & $\mathrm{M}$ & $\mathrm{n} / \mathrm{a}$ & $\mathrm{L}$ \\
\hline Park, 2016 [65] & $\mathrm{L}$ & $\mathrm{H}$ & $\mathrm{L}$ & $\mathrm{M}$ & $\mathrm{L}$ & M & $\mathrm{M}$ \\
\hline Penel, 2008 [66] & $\bar{L}$ & $\mathrm{H}$ & $\mathrm{M}$ & $\mathrm{L}$ & $\mathrm{M}$ & M & $\mathrm{H}$ \\
\hline Penel, 2008 [67] & $\mathrm{L}$ & $\mathrm{H}$ & $\mathrm{L}$ & $\mathrm{L}$ & M & $\mathrm{n} / \mathrm{a}$ & M \\
\hline Pinato, 2015 [68] & $\mathrm{L}$ & $\mathrm{H}$ & $M$ & $\mathrm{~L}$ & $\mathrm{~L}$ & $\mathrm{~L}$ & M \\
\hline Pointillart, 2011 [69] & $\mathrm{L}$ & $\mathrm{H}$ & $\mathrm{L}$ & $\mathrm{L}$ & M & $\mathrm{n} / \mathrm{a}$ & M \\
\hline $\begin{array}{c}\text { Roychowdhury, } \\
2003 \text { [70] }\end{array}$ & $\mathrm{L}$ & $\mathrm{H}$ & $\mathrm{L}$ & M & $\mathrm{M}$ & $\mathrm{n} / \mathrm{a}$ & M \\
\hline Rydzek, 2015 [71] & $\mathrm{L}$ & $\mathrm{H}$ & $\mathrm{L}$ & M & M & $\mathrm{n} / \mathrm{a}$ & M \\
\hline Schoenfeld, 2020 [72] & $\mathrm{L}$ & $\mathrm{H}$ & $\mathrm{L}$ & $\mathrm{L}$ & $\mathrm{L}$ & $\mathrm{n} / \mathrm{a}$ & $\mathrm{L}$ \\
\hline Scott, 2002 [73] & $\mathrm{L}$ & $\mathrm{L}$ & $\mathrm{L}$ & $\mathrm{L}$ & $\mathrm{L}$ & $\mathrm{n} / \mathrm{a}$ & $\mathrm{L}$ \\
\hline Seow, 2013 [74] & $\mathrm{L}$ & $\mathrm{H}$ & $\mathrm{L}$ & $\mathrm{L}$ & $\mathrm{M}$ & $\mathrm{n} / \mathrm{a}$ & $\mathrm{M}$ \\
\hline Shen, 2007 [75] & $\mathrm{L}$ & $\mathrm{H}$ & $\mathrm{L}$ & $\mathrm{L}$ & $\mathrm{L}$ & $\mathrm{n} / \mathrm{a}$ & $\mathrm{L}$ \\
\hline Soubeyran, 2012 [76] & $\mathrm{L}$ & $\mathrm{H}$ & $\mathrm{L}$ & $\mathrm{L}$ & $\mathrm{H}$ & $\mathrm{n} / \mathrm{a}$ & M \\
\hline Sutradhar, 2014 [77] & $\mathrm{L}$ & $\mathrm{H}$ & M & $\mathrm{L}$ & $\mathrm{L}$ & M & M \\
\hline Suzuki, 2020 [78] & $\mathrm{L}$ & $\mathrm{H}$ & M & $\mathrm{L}$ & $\mathrm{L}$ & $\mathrm{n} / \mathrm{a}$ & M \\
\hline Tripodoro, 2019 [80] & $\mathrm{L}$ & $\mathrm{H}$ & $\mathrm{L}$ & $\mathrm{L}$ & M & $\mathrm{n} / \mathrm{a}$ & M \\
\hline Tsai, 2014 [79] & $\mathrm{L}$ & M & $\mathrm{L}$ & $\mathrm{L}$ & $\mathrm{L}$ & $\mathrm{n} / \mathrm{a}$ & $\mathrm{L}$ \\
\hline Ueno, $2000[81]$ & $\mathrm{L}$ & $\mathrm{H}$ & $\mathrm{L}$ & $\mathrm{L}$ & $\mathrm{L}$ & $\mathrm{n} / \mathrm{a}$ & $\mathrm{L}$ \\
\hline $\begin{array}{c}\text { van der Linden, } \\
2005 \text { [82] }\end{array}$ & $\mathrm{L}$ & $\mathrm{M}$ & $\mathrm{L}$ & $\mathrm{L}$ & $\mathrm{M}$ & $\mathrm{n} / \mathrm{a}$ & $\mathrm{L}$ \\
\hline Vigano, 2000 [83] & $\mathrm{L}$ & M & $\mathrm{L}$ & $\mathrm{L}$ & $\mathrm{L}$ & $\mathrm{n} / \mathrm{a}$ & $\mathrm{L}$ \\
\hline Villa, 2011 [84] & $\mathrm{L}$ & $\mathrm{M}$ & M & $\mathrm{L}$ & $\mathrm{L}$ & $\mathrm{M}$ & $\mathrm{M}$ \\
\hline Wei, 2019 [85] & $\mathrm{L}$ & M & M & M & M & $\mathrm{n} / \mathrm{a}$ & M \\
\hline Yamashita, 2011 [86] & $\bar{L}$ & $\mathrm{H}$ & $\mathrm{L}$ & $\mathrm{L}$ & $\mathrm{L}$ & $\mathrm{n} / \mathrm{a}$ & $\mathrm{L}$ \\
\hline
\end{tabular}

H: high risk-of-bias; L: low risk-of-bias; M: moderate risk-of-bias; n/a: not applicable. 
Table 3. Summary of the prediction models.

\begin{tabular}{|c|c|c|c|c|c|c|c|c|c|c|c|c|c|c|c|c|c|c|c|c|c|c|}
\hline \multicolumn{23}{|c|}{ Variables } \\
\hline Study & FU & $\mathbf{N}$ & $\begin{array}{l}\text { Name } \\
\text { Model }\end{array}$ & $\begin{array}{l}\text { Cancer } \\
\text { Type }\end{array}$ & 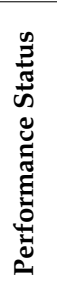 & 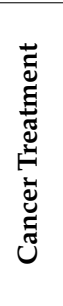 & $\stackrel{\times}{凶}$ & 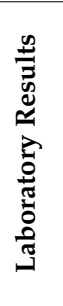 & 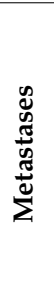 & 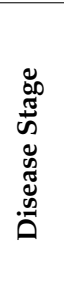 & $\underset{<}{\stackrel{8}{4}}$ & 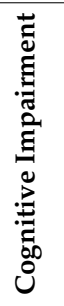 & 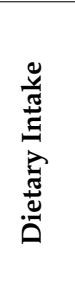 & 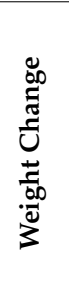 & 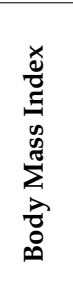 & 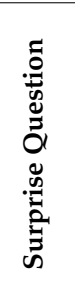 & 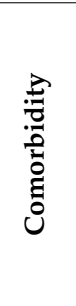 & 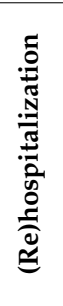 & 岂 & Discriminative Ability & Calibration & $\begin{array}{c}\text { External } \\
\text { Validation }\end{array}$ \\
\hline Brunello, 2016 [21] & 12 & 658 & Onco-MPI & * & $*$ & & * & & & $*$ & * & * & & & * & & & & $\begin{array}{l}* \\
* \\
*\end{array}$ & $\begin{array}{c}\text { c-statistic } 0.869 \\
(95 \% \text { CI } 0.841-0.897)\end{array}$ & Good & $\mathrm{n} / \mathrm{a}$ \\
\hline \multirow[t]{3}{*}{ Cesari [22] } & 12 & 200 & IADL & & & & & & & & & & & & & & & & * & $\begin{array}{c}\text { AUC 0.676 } \\
(95 \% \text { CI } 0.532-0.821)\end{array}$ & $\mathrm{n} / \mathrm{a}$ & $\mathrm{n} / \mathrm{a}$ \\
\hline & & & SPPB & & & & & & & & & & & & & & & & * & $\begin{array}{c}\text { AUC } 0.638 \\
(95 \% \text { CI } 0.483-0.792)\end{array}$ & $\mathrm{n} / \mathrm{a}$ & $\mathrm{n} / \mathrm{a}$ \\
\hline & & & UGS & & & & & & & & & & & & & & & & * & $\begin{array}{c}\text { AUC } 0.686 \\
(95 \% \text { CI } 0.560-0.812)\end{array}$ & $\mathrm{n} / \mathrm{a}$ & $\mathrm{n} / \mathrm{a}$ \\
\hline Chow, 2008 [24] & 12 & 395 & $\mathrm{n} / \mathrm{a}$ & $*$ & * & & & & * & & & & & & & & & & & c-statistic 0.66 & $\mathrm{n} / \mathrm{a}$ & $\begin{array}{c}N=467 \\
\text { c-statistic } \\
0.63\end{array}$ \\
\hline Deans, 2007 [28] & 24 & 220 & $\mathrm{u} / \mathrm{a}$ & & $*$ & & & $*$ & & $*$ & & & & $*$ & & & & & & AUC 0.85 & $\mathrm{n} / \mathrm{a}$ & $\mathrm{n} / \mathrm{a}$ \\
\hline Giantin, 2013 [36] & 12 & 160 & MPI & * & & * & & & & & & * & & & & & & & $\begin{array}{l}* \\
* \\
*\end{array}$ & $\begin{array}{c}\text { AUC 0.874 } \\
(95 \% \text { CI } 0.819-0.928)\end{array}$ & $\mathrm{n} / \mathrm{a}$ & $\mathrm{n} / \mathrm{a}$ \\
\hline \multirow[t]{3}{*}{ Jang, 2014 [47] } & $\mathrm{n} / \mathrm{a}$ & 1655 & KPS & & * & & & & & & & & & & & & & & & c-statistic 0.63 & $\mathrm{n} / \mathrm{a}$ & $\mathrm{n} / \mathrm{a}$ \\
\hline & & & PPS & & * & & & & & & & & & & & & & & & c-statistic 0.63 & $\mathrm{n} / \mathrm{a}$ & $\mathrm{n} / \mathrm{a}$ \\
\hline & & & ECOG & & * & & & & & & & & & & & & & & & c-statistic 0.64 & $\mathrm{n} / \mathrm{a}$ & $\mathrm{n} / \mathrm{a}$ \\
\hline Jonna, 2016 [48] & 12 & 803 & $\mathrm{n} / \mathrm{a}$ & * & & * & * & & & & & * & & & * & & & * & * & $\begin{array}{c}\text { c-statistic } 0.66 \\
(95 \% \text { CI } 0.58-0.72)\end{array}$ & $\mathrm{n} / \mathrm{a}$ & $\mathrm{n} / \mathrm{a}$ \\
\hline
\end{tabular}


Table 3. Cont.

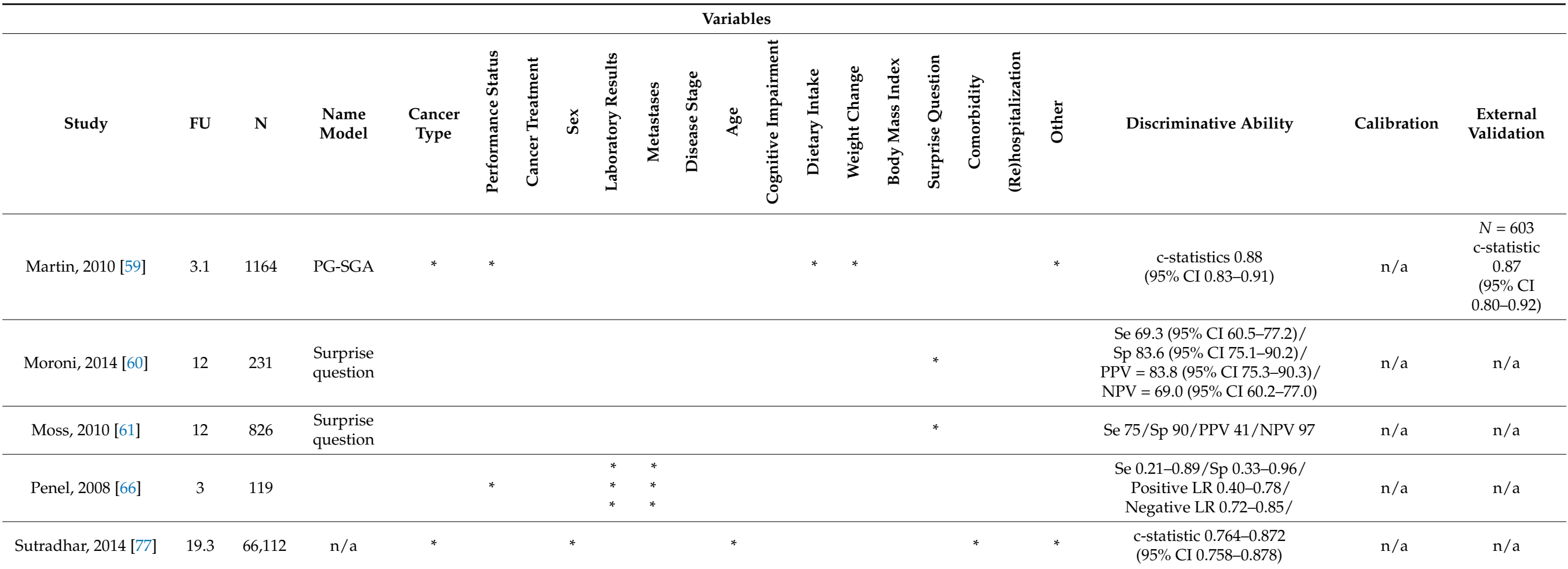

ECOG: Eastern Cooperative Oncology Group; FU: follow-up; KPS: IADL: instrumental activities of daily living; Karnofsky Performance Status; LR: likelihood ratio; MPI: multidimensional prognostic index; n/a: not available; NPV: negative predictive value; Onco-MPI: Oncological-multidimensional prognostic index; PG-SGA: Patient-generated Subjective Global Assessment; PPS: Palliative Performance Scale; PPV: positive predictive value; Se: sensitivity; Sp: specificity; SPPB: Short Physical Performance Battery; UGS: usual gait speed. Legend: an asterisk represents the presence of the variable, whereby two or more asterisks per variable represent different subcategories. 


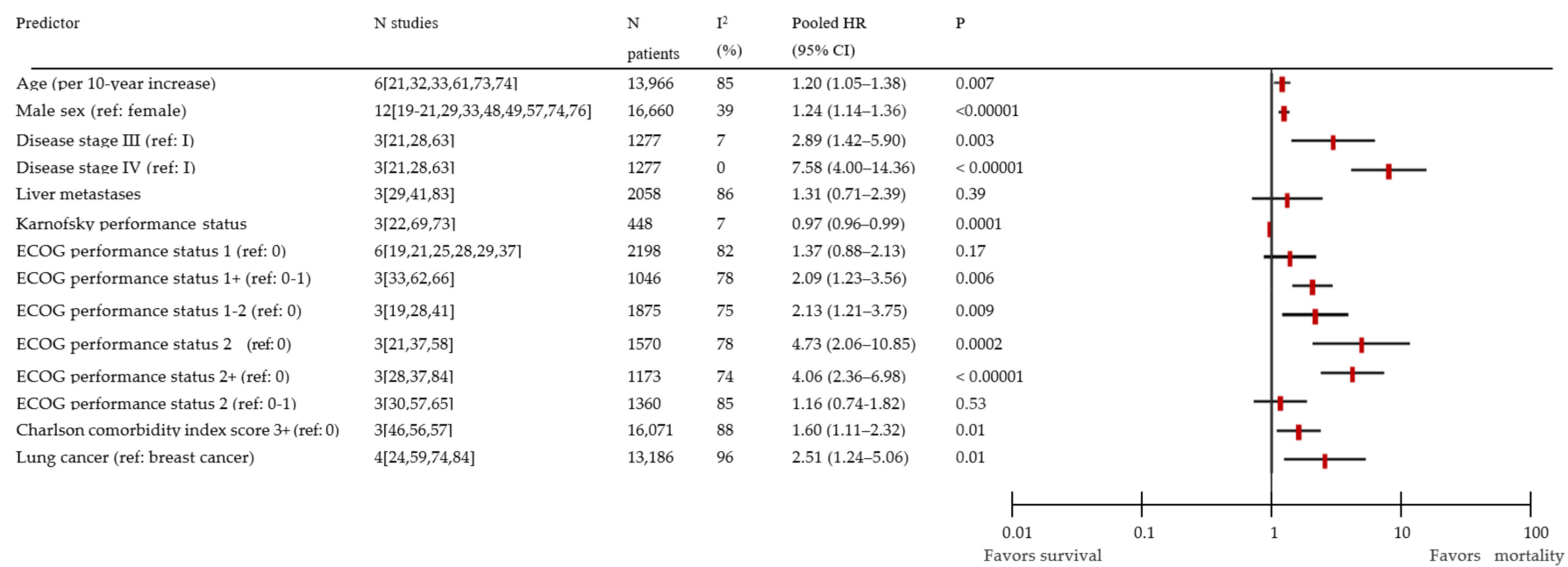

Figure 3. Forest plot of pooled hazard ratios for mortality with a random-effects model. CI: confidence interval; HR: hazard ratio; ECOG: Eastern Cooperative Oncology Group.

\section{Discussion}

We aimed to identify significant predictors of mortality within a period 3-24 months for patients with advanced cancer in published literature. Overall, the predictors we found can be categorized into three main groups: the surprise question; clinical variables (age, male sex, disease stage, performance status, comorbidity, cognitive impairment, (sub)cutaneous metastases, and lung cancer); and laboratory variables (serum albumin and hemoglobin). Almost all predictors were non-tumor specific and may improve the identification of cancer patients at risk of death in the set time period. Better identification might enable conversations on advance care planning. However, the usefulness of some predictors in the clinical setting may be questionable. It might seem obvious that an advanced disease stage (III or IV) compared to a localized stage (I) is associated with mortality. Considering hemoglobin, it is unknown whether chemotherapy-induced or tumor-induced anemia were specifically considered, although this may be relevant. Furthermore, the identified predictors might not be exclusively associated with mortality within 3-24 months. For example, the performance status of a patient is, on the one hand, included in indexes to predict the terminal phase, such as in the Palliative Prognostic Score, the Palliative Prognostic Index, and the Chuang Prognostic Score [87]. On the other hand, the performance status is also included in tumor-specific indexes to predict mortality within a longer time frame exceeding 24 months [88-90].

The advantage of non-tumor specific predictors is that they apply to patients with various types of cancer. Furthermore, those predictors could be used by both medical specialists and other physicians, such as general practitioners. In theory, studies that were conducted in heterogeneous groups of patients, i.e., patients with various types of cancer, comorbidity and other characteristics, were most useful for our study. However, approximately half of the studies included patients with a specific type of cancer or a specific complication, as for example, bone metastases. To find non-tumor specific predictors in the meta-analysis, we only included predictors that were examined in heterogeneous study populations or in at least two groups of patients with different types of cancer. As a result, we observed substantial heterogeneity across studies in the meta-analysis for the predictors: age, the different variants of ECOG performance status, Charlson comorbidity index, lung cancer, and liver metastasis. However, the observed heterogeneity may also have been caused by different follow-up periods in the separate studies, different mortality rates, and differences between studies regarding the percentage of patients with locally advanced vs metastatic disease. Therefore, the substantial heterogeneity we found for the mentioned predictors can be expected. For example, the presence of liver metastasis in a patient with stomach cancer probably has a worse prognosis than in a patient with breast cancer. In 
addition, a patient with a poor performance status might seem to have a worse prognosis when examining a period of 24 months compared to a period of six months. Nevertheless, although heterogeneity was found between studies, we found various generic predictors of mortality. To optimize the prediction of mortality in a heterogeneous cancer population, multiple predictors must be considered together. We recommend the examination of several predictors in a prospective study in a heterogeneous cancer population.

Our study demonstrates the clinical importance of the surprise question, which was one of the most significant predictors in the meta-analysis. However, this finding should be interpreted with some caution because the surprise question was examined in only two studies. Furthermore, it is important to bear in mind that the surprise question has an aspect of subjectivity because it relates to the physician's opinion [91]. Therefore, the generalizability of the surprise question might be questioned. Despite this, the surprise question is commonly used in the identification of patients with palliative care needs and included in palliative care needs assessment tools, such as the RADboud indicators for Palliative Care needs (RADPAC), Supportive and Palliative Care Indicators Tool (SPICT), and Prognostic Indicator Guide (PIG) [92]. Despite the fact that the surprise question is widely believed to be useful, there is abundant room for discussion concerning whether it should be used as a predictor of mortality, possibly combined with other variables, or only included in the assessment of palliative care needs [93,94].

Remarkably, apart from the surprise question, all other prediction models consisted of different variables or sets of variables. Their discriminative ability was mostly moderate, and some were good. However, a note of caution is due in the applicability of almost all the models since their calibration, which shows observed and predicted risk of mortality, was not studied. Another source of caution is the lack of external validation of the models. Despite the possible limitations of the models, two models with a good discriminative ability might be interesting for further study: the Patient-generated Subjective Global Assessment, which was externally validated; and the Oncological-multidimensional prognostic index, which had a good calibration. Nonetheless, there is a definite need for a general, high-quality prediction model for the accurate prediction of mortality in patients with advanced cancer.

This study had some limitations. Firstly, because we performed a meta-analysis, some predictors with slight differences in measuring units or cut-offs could not be pooled. An example of such a predictor was weight loss, which was studied at various cut-offs of kilograms. Therefore, the number of pooled studies for each predictor was small, e.g., the inclusion of two studies, which might limit the strength of a meta-analysis. Secondly, studies with moderate or high risk-of-bias scored poorly on the study attrition of our modified QUIPS tool. Lastly, the inclusion of published studies, exclusively from 2000 onward, could have led to the exclusion of some predictors of mortality.

\section{Conclusions}

The surprise question and general clinical (age, male sex, disease stage, performance status, comorbidity, cognitive impairment, (sub)cutaneous metastases, and lung cancer) and laboratory variables (serum albumin and hemoglobin) are non-tumor specific predictors of mortality within 3-24 months in patients with advanced cancer. Physicians could apply those predictors of mortality within 3-24 months in the timely initiation of advance care planning. Due to the lack of high-quality prediction models, the identified predictors can provide guidance in selecting candidate predictors for future prospective studies, which will explore the development of a general prediction model for mortality. Furthermore, future research should also examine the association of using such a prediction model with the timely initiation of advance care planning.

Supplementary Materials: The following are available online at https:/ / www.mdpi.com/article / 10.3390/cancers14020328/s1, Table S1: Search strategy for the online databases, Table S2: Domains and items for risk-of-bias assessment, Table S3: Hazard ratios for mortality based on studies with 
low risk-of-bias, Table S4: Variables not included in the meta-analysis, Figure S1: Forest plots per individual predictor using random-effects model, Figure S2: Funnel plots.

Author Contributions: C.O., S.A.D., A.v.d.H. and C.C.D.v.d.R. developed the concept and study design. C.O. and S.A.D. acquired data. C.O., S.A.D. and D.N. evaluated and analyzed the data. C.O. drafted the initial manuscript. The corresponding author had full access to all the data in the study and had final responsibility for the decision to submit for publication. All authors have read and agreed to the published version of the manuscript.

Funding: This research was funded by the Netherlands Organization for Health Research and Development (ZonMw) [grant number 844001209].

Acknowledgments: We thank researcher Hester F. Lingsma for her assistance in designing the study. We also thank Gerdien B. de Jonge, Biomedical Information Specialist at the Erasmus MC Medical Library, for her assistance with the systematic search.

Conflicts of Interest: The authors declare no conflict of interest. The funders had no role in the design of the study; in the collection, analyses, or interpretation of data; in the writing of the manuscript, or in the decision to publish the results. The corresponding author had full access to all the data in the study and had final responsibility for the decision to submit for publication.

\section{References}

1. Rietjens, J.A.C.; Sudore, R.L.; Connolly, M.; van Delden, J.J.; Drickamer, M.A.; Droger, M.; van der Heide, A.; Heyland, D.K.; Houttekier, D.; Janssen, D.J.A.; et al. Definition and recommendations for advance care planning: An international consensus supported by the European Association for Palliative Care. Lancet Oncol. 2017, 18, e543-e551. [CrossRef]

2. Murray, A.S.; Kendall, M.; Boyd, K.; Sheikh, A. Illness trajectories and palliative care. BMJ 2005, 330, 1007-1011. [CrossRef]

3. National Institute for Health and Care Excellence. End of Life Care for Adults. Available online: https://www.nice.org.uk/ guidance/ qs13 (accessed on 6 January 2022).

4. NSW Health. End of Life and Palliative Care Framework 2019-2024. Available online: https://www.health.nsw.gov.au/ palliativecare/Publications / eol-pc-framework.pdf (accessed on 6 January 2022).

5. IKNL. Netherlands Quality Framework for Palliative Care. Available online: https://pznlsawebprod.blob.core.windows.net/ mediacontainer/pznl/media/themabestanden/kwaliteitskader\%20palliatieve\%20zorg\%20nederland/netherlands-qualityframework-for-palliative-care.pdf (accessed on 6 January 2022).

6. Pattison, M.; Romer, A.L. Improving Care Through the End of Life: Launching a Primary Care Clinic-Based Program. J. Palliat. Med. 2001, 4, 249-254. [CrossRef]

7. White, N.; Kupeli, N.; Vickerstaff, V.; Stone, P. How accurate is the 'Surprise Question' at identifying patients at the end of life? A systematic review and meta-analysis. BMC Med. 2017, 15, 139. [CrossRef]

8. Simmons, C.P.; McMillan, D.; McWilliams, K.; Sande, T.A.; Fearon, K.C.; Tuck, S.; Fallon, M.; Laird, B.J. Prognostic Tools in Patients with Advanced Cancer: A Systematic Review. J. Pain Symptom Manag. 2017, 53, 962-970. [CrossRef] [PubMed]

9. Bramer, W.; Milic, J.; Mast, F. Reviewing retrieved references for inclusion in systematic reviews using EndNote. J. Med. Libr. Assoc. 2017, 105, 84-87. [CrossRef]

10. Moons, K.G.M.; de Groot, J.A.H.; Bouwmeester, W.; Vergouwe, Y.; Mallett, S.; Altman, D.G.; Reitsma, J.B.; Collins, G.S. Critical Appraisal and Data Extraction for Systematic Reviews of Prediction Modelling Studies: The CHARMS Checklist. PLoS Med. 2014, 11, e1001744. [CrossRef] [PubMed]

11. Hayden, J.A.; Côté, P.; Bombardier, C. Evaluation of the Quality of Prognosis Studies in Systematic Reviews. Ann. Intern. Med. 2006, 144, 427-437. [CrossRef] [PubMed]

12. Page, M.J.; McKenzie, J.E.; Bossuyt, P.M.; Boutron, I.; Hoffmann, T.C.; Mulrow, C.D.; Shamseer, L.; Tetzlaff, J.M.; Akl, E.A.; Brennan, S.E.; et al. The PRISMA 2020 statement: An updated guideline for reporting systematic reviews. BMJ 2021, $372, \mathrm{n} 71$. [CrossRef]

13. Tierney, J.F.; Stewart, L.A.; Ghersi, D.; Burdett, S.; Sydes, M.R. Practical methods for incorporating summary time-to-event data into meta-analysis. Trials 2007, 8, 16. [CrossRef] [PubMed]

14. Higgins, J.P.T.; Thompson, S.G. Quantifying heterogeneity in a meta-analysis. Stat. Med. 2002, 21, 1539-1558. [CrossRef]

15. OncologyPRO. 2020. Available online: https://oncologypro.esmo.org/oncology-in-practice/practice-tools/performance-scales (accessed on 6 January 2022).

16. Grunkemeier, G.L.; Jin, R. Receiver operating characteristic curve analysis of clinical risk models. Ann. Thorac. Surg. 2001, 72, 323-326. [CrossRef]

17. Steyerberg, E.W.; Vergouwe, Y. Towards better clinical prediction models: Seven steps for development and an ABCD for validation. Eur. Heart J. 2014, 35, 1925-1931. [CrossRef]

18. Egger, M.; Smith, G.D.; Schneider, M.; Minder, C. Bias in meta-analysis detected by a simple, graphical test. BMJ 1997, 315, 629-634. [CrossRef] 
19. Bartels, R.H.M.A.; Feuth, T.; van der Maazen, R.; Verbeek, A.L.M.; Kappelle, A.C.; Grotenhuis, J.A.; Leer, J.W. Development of a model with which to predict the life expectancy of patients with spinal epidural metastasis. Cancer 2007, 110, 2042-2049. [CrossRef]

20. Braun, D.P.; Gupta, D.; Staren, E.D. Quality of life assessment as a predictor of survival in non-small cell lung cancer. BMC Cancer 2011, 11, 353. [CrossRef] [PubMed]

21. Brunello, A.; Fontana, A.; Zafferri, V.; Panza, F.; Fiduccia, P.; Basso, U.; Copetti, M.; Lonardi, S.; Roma, A.; Falci, C.; et al. Development of an oncological-multidimensional prognostic index (Onco-MPI) for mortality prediction in older cancer patients. J. Cancer Res. Clin. Oncol. 2016, 142, 1069-1077. [CrossRef]

22. Cesari, M.; Cerullo, F.; Zamboni, V.; di Palma, R.; Scambia, G.; Balducci, L.; Incalzi, R.A.; Vellas, B.; Gambassi, G. Functional Status and Mortality in Older Women with Gynecological Cancer. J. Gerontol. Ser. A Biol. Sci. Med. Sci. 2013, 68, 1129-1133. [CrossRef]

23. Chen, C.; Feng, C.; Yu, C.; Peng, C.; Lai, H.; Cheng, K.; Lin, Y.; Huang, W.; Kao, J. Prognostic factors associated with the survival of patients with gastric adenocarcinoma: A retrospective study. Adv. Dig. Med. 2019, 7, 68-76. [CrossRef]

24. Chow, E.; Abdolell, M.; Panzarella, T.; Harris, K.; Bezjak, A.; Warde, P.; Tannock, I. Predictive Model for Survival in Patients with Advanced Cancer. J. Clin. Oncol. 2008, 26, 5863-5869. [CrossRef] [PubMed]

25. Collette, L.; van Andel, G.; Bottomley, A.; Oosterhof, G.O.; Albrecht, W.; de Reijke, T.M.; Fossà, S.D. Is Baseline Quality of Life Useful for Predicting Survival with Hormone-Refractory Prostate Cancer? A Pooled Analysis of Three Studies of the European Organisation for Research and Treatment of Cancer Genitourinary Group. J. Clin. Oncol. 2004, 22, 3877-3885. [CrossRef] [PubMed]

26. Collins, A.; Sundararajan, V.; Brand, C.A.; Moore, G.; Lethborg, C.; Gold, M.; Murphy, M.A.; Bohensky, M.A.; Philip, J. Clinical presentation and patterns of care for short-term survivors of malignant glioma. J. Neuro-Oncol. 2014, 119, 333-341. [CrossRef]

27. Contreras-Bolívar, V.; Sánchez-Torralvo, F.J.; Ruiz-Vico, M.; González-Almendros, I.; Barrios, M.; Padín, S.; Alba, E.; Olveira, G. GLIM Criteria Using Hand Grip Strength Adequately Predict Six-Month Mortality in Cancer Inpatients. Nutrients 2019, 11, 2043. [CrossRef] [PubMed]

28. Deans, D.A.C.; Wigmore, S.J.; de Beaux, A.C.; Paterson-Brown, S.; Garden, O.J.; Fearon, K.C.H. Clinical prognostic scoring system to aid decision-making in gastro-oesophageal cancer. BJS 2007, 94, 1501-1508. [CrossRef] [PubMed]

29. Dharma-Wardene, M.; Au, H.-J.; Hanson, J.; Dupere, D.; Hewitt, J.; Feeny, D. Baseline FACT-G score is a predictor of survival for advanced lung cancer. Qual. Life Res. 2004, 13, 1209-1216. [CrossRef]

30. Efficace, F.; Bottomley, A.; Smit, E.F.; Lianes, P.; Legrand, C.; Debruyne, C.; Schramel, F.; Smit, H.J.; Gaafar, R.; Biesma, B.; et al Is a patient's self-reported health-related quality of life a prognostic factor for survival in non-small-cell lung cancer patients? A multivariate analysis of prognostic factors of EORTC study 08975. Ann. Oncol. 2006, 17, 1698-1704. [CrossRef]

31. Ferrigno, D.; Buccheri, G.; Ricca, I. Prognostic significance of blood coagulation tests in lung cancer. Eur. Respir. J. 2001, 17, 667-673. [CrossRef] [PubMed]

32. Fielding, R.; Wong, W.S. Quality of life as a predictor of cancer survival among Chinese liver and lung cancer patients. Eur. J. Cancer 2007, 43, 1723-1730. [CrossRef] [PubMed]

33. Filippini, G.; Falcone, C.; Boiardi, A.; Broggi, G.; Bruzzone, M.G.; Caldiroli, D.; Farina, R.; Farinotti, M.; Fariselli, L.; Finocchiaro, G.; et al. Prognostic factors for survival in 676 consecutive patients with newly diagnosed primary glioblastoma. Neuro-Oncology 2008, 10, 79-87. [CrossRef]

34. Gagnon, B.; Agulnik, J.S.; Gioulbasanis, I.; Kasymjanova, G.; Morris, D.; Macdonald, N. Montreal prognostic score: Estimating survival of patients with non-small cell lung cancer using clinical biomarkers. Br. J. Cancer 2013, 109, 2066-2071. [CrossRef]

35. Geraci, J.M.; Tsang, W.; Valdres, R.V.; Escalante, C.P. Progressive disease in patients with cancer presenting to an emergency room with acute symptoms predicts short-term mortality. Support. Care Cancer 2006, 14, 1038-1045. [CrossRef] [PubMed]

36. Giantin, V.; Valentini, E.; Iasevoli, M.; Falci, C.; Siviero, P.; de Luca, E.; Maggi, S.; Martella, B.; Orrù, G.; Crepaldi, G.; et al. Does the Multidimensional Prognostic Index (MPI), based on a Comprehensive Geriatric Assessment (CGA), predict mortality in cancer patients? Results of a prospective observational trial. J. Geriatr. Oncol. 2013, 4, 208-217. [CrossRef] [PubMed]

37. Griguolo, G.; Jacot, W.; Kantelhardt, E.; Dieci, M.V.; Bourgier, C.; Thomssen, C.; Bailleux, C.; Miglietta, F.; Braccini, A.-L.; Conte, P.; et al. External validation of Modified Breast Graded Prognostic Assessment for breast cancer patients with brain metastases: A multicentric European experience. Breast 2018, 37, 36-41. [CrossRef]

38. Gripp, S.; Moeller, S.; Bölke, E.; Schmitt, G.; Matuschek, C.; Asgari, S.; Asgharzadeh, F.; Roth, S.; Budach, W.; Franz, M.; et al Survival Prediction in Terminally Ill Cancer Patients by Clinical Estimates, Laboratory Tests, and Self-Rated Anxiety and Depression. J. Clin. Oncol. 2007, 25, 3313-3320. [CrossRef]

39. Gupta, D.; Lis, C.G.; Dahlk, S.L.; Vashi, P.G.; Grutsch, J.F.; Lammersfeld, C.A. Bioelectrical impedance phase angle as a prognostic indicator in advanced pancreatic cancer. Br. J. Nutr. 2004, 92, 957-962. [CrossRef] [PubMed]

40. Gupta, D.; Lammersfeld, C.A.; Vashi, P.G.; King, J.; Dahlk, S.L.; Grutsch, J.F.; Lis, C.G. Bioelectrical impedance phase angle in clinical practice: Implications for prognosis in stage IIIB and IV non-small cell lung cancer. BMC Cancer 2009, 9, 37. [CrossRef] [PubMed]

41. Hoang, T.; Xu, R.; Schiller, J.H.; Bonomi, P.; Johnson, D.H. Clinical Model to Predict Survival in Chemonaive Patients with Advanced Non-Small-Cell Lung Cancer Treated With Third-Generation Chemotherapy Regimens Based on Eastern Cooperative Oncology Group Data. J. Clin. Oncol. 2005, 23, 175-183. [CrossRef] [PubMed] 
42. Hong, Y.-F.; Chen, Z.-H.; Ma, X.-K.; Li, X.; Wu, D.-H.; Chen, J.; Dong, M.; Wei, L.; Wang, T.-T.; Ruan, D.-Y.; et al. Comparison of five models for end-stage liver disease in predicting the survival rate of patients with advanced hepatocellular carcinoma. Tumor Biol. 2015, 37, 5265-5273. [CrossRef]

43. Hosono, N.; Ueda, T.; Tamura, D.; Aoki, Y.; Yoshikawa, H. Prognostic Relevance of Clinical Symptoms in Patients with Spinal Metastases. Clin. Orthop. Relat. Res. 2005, 436, 196-201. [CrossRef]

44. Hui, D.; Bansal, S.; Morgado, M.; Dev, R.; Chisholm, G.; Bruera, E. Phase angle for prognostication of survival in patients with advanced cancer: Preliminary findings. Cancer 2014, 120, 2207-2214. [CrossRef]

45. Hui, D.; Park, M.; Liu, D.; Paiva, C.E.; Suh, S.-Y.; Morita, T.; Bruera, E. Clinician prediction of survival versus the Palliative Prognostic Score: Which approach is more accurate? Eur. J. Cancer 2016, 64, 89-95. [CrossRef] [PubMed]

46. Iversen, L.H.; Nørgaard, M.; Jacobsen, J.; Laurberg, S.; Sørensen, H.T. The Impact of Comorbidity on Survival of Danish Colorectal Cancer Patients from 1995 to 2006-A Population-Based Cohort Study. Dis. Colon Rectum 2009, 52, 71-78. [CrossRef]

47. Jang, R.W.; Caraiscos, V.B.; Swami, N.; Banerjee, S.; Mak, E.; Kaya, E.; Rodin, G.; Bryson, J.; Ridley, J.Z.; Le, L.W.; et al. Simple Prognostic Model for Patients with Advanced Cancer Based on Performance Status. J. Oncol. Pr. 2014, 10, e335-e341. [CrossRef] [PubMed]

48. Jonna, S.; Chiang, L.; Liu, J.; Carroll, M.B.; Flood, K.; Wildes, T.M. Geriatric assessment factors are associated with mortality after hospitalization in older adults with cancer. Support. Care Cancer 2016, 24, 4807-4813. [CrossRef] [PubMed]

49. Katagiri, H.; Takahashi, M.; Wakai, K.; Sugiura, H.; Kataoka, T.; Nakanishi, K. Prognostic factors and a scoring system for patients with skeletal metastasis. J. Bone Jt. Surgery. Br. Vol. 2005, 87, 698-703. [CrossRef] [PubMed]

50. Kilgour, R.D.; Vigano, A.; Trutschnigg, B.; Lucar, E.; Borod, M.; Morais, J.A. Handgrip strength predicts survival and is associated with markers of clinical and functional outcomes in advanced cancer patients. Support. Care Cancer 2013, 21, 3261-3270. [CrossRef]

51. Kim, J.H.; Kim, J.H.; Choi, J.H.; Kim, C.H.; Jung, Y.K.; Yim, H.J.; Yeon, J.E.; Park, J.-J.; Kim, J.S.; Bak, Y.-T.; et al. Value of the model for end-stage liver disease for predicting survival in hepatocellular carcinoma patients treated with transarterial chemoembolization. Scand. J. Gastroenterol. 2009, 44, 346-357. [CrossRef]

52. Kinoshita, A.; Onoda, H.; Takano, K.; Imai, N.; Saeki, C.; Fushiya, N.; Miyakawa, Y.; Nishino, H.; Tajiri, H. Pretreatment serum C-reactive protein level predicts poor prognosis in patients with hepatocellular carcinoma. Med. Oncol. 2012, 29, 2800-2808. [CrossRef]

53. Langendijk, H.; Aaronson, N.K.; de Jong, J.M.; Velde, G.P.T.; Muller, M.J.; Wouters, M. The prognostic impact of quality of life assessed with the EORTC QLQ-C30 in inoperable non-small cell lung carcinoma treated with radiotherapy. Radiother. Oncol. 2000, 55, 19-25. [CrossRef]

54. Liljehult, M.M.; Buus, L.; Liljehult, J.M.; Rasmussen, B.K. Walking ability in patients with glioblastoma: Prognostic value of the Berg Balance Scale and the 10 meter walk test. J. Neuro-Oncol. 2017, 135, 335-342. [CrossRef]

55. Limquiaco, J.L.; Wong, G.L.; Wong, V.W.; Lai, P.B.; Chan, H.L. Evaluation of Model for End Stage Liver Disease (MELD)-based systems as prognostic index for hepatocellular carcinoma. J. Gastroenterol. Hepatol. 2009, 24, 63-69. [CrossRef] [PubMed]

56. Lund, L.; Jacobsen, J.; Nørgaard, M.; McLaughlin, J.K.; Blot, W.J.; Borre, M.; Sørensen, H.T. The Prognostic Impact of Comorbidities on Renal Cancer, 1995 to 2006: A Danish Population Based Study. J. Urol. 2009, 182, 35-40. [CrossRef] [PubMed]

57. Maione, P.; Perrone, F.; Gallo, C.; Manzione, L.; Piantedosi, F.; Barbera, S.; Cigolari, S.; Rosetti, F.; Piazza, E.; Robbiati, S.F.; et al Pretreatment Quality of Life and Functional Status Assessment Significantly Predict Survival of Elderly Patients with Advanced Non-Small-Cell Lung Cancer Receiving Chemotherapy: A Prognostic Analysis of the Multicenter Italian Lung Cancer in the Elderly Study. J. Clin. Oncol. 2005, 23, 6865-6872. [CrossRef] [PubMed]

58. Marrero, J.A.; Fontana, R.J.; Barrat, A.; Askari, F.K.; Conjeevaram, H.S.; Su, G.; Lok, A.S.-F. Prognosis of hepatocellular carcinoma: Comparison of 7 staging systems in an American cohort. Hepatology 2005, 41, 707-715. [CrossRef] [PubMed]

59. Martin, L.; Watanabe, S.; Fainsinger, R.; Lau, F.; Ghosh, S.; Quan, H.; Atkins, M.; Fassbender, K.; Downing, G.M.; Baracos, V. Prognostic Factors in Patients with Advanced Cancer: Use of the Patient-Generated Subjective Global Assessment in Survival Prediction. J. Clin. Oncol. 2010, 28, 4376-4383. [CrossRef]

60. Moroni, M.; Zocchi, D.; Bolognesi, D.; Abernethy, A.; Rondelli, R.; Savorani, G.; Salera, M.; Dall'Olio, F.G.; Galli, G.; Biasco, G.; et al. The 'surprise' question in advanced cancer patients: A prospective study among general practitioners. Palliat. Med. 2014, 28, 959-964. [CrossRef]

61. Moss, A.H.; Lunney, J.R.; Culp, S.; Auber, M.; Kurian, S.; Rogers, J.; Dower, J.; Abraham, J. Prognostic significance of the "surprise" question in cancer patients. J. Palliat. Med. 2010, 13, 837-840. [CrossRef]

62. Motzer, R.J.; Bacik, J.; Schwartz, L.H.; Reuter, V.; Russo, P.; Marion, S.; Mazumdar, M. Prognostic Factors for Survival in Previously Treated Patients with Metastatic Renal Cell Carcinoma. J. Clin. Oncol. 2004, 22, 454-463. [CrossRef]

63. Norman, K.; Stobäus, N.; Zocher, D.; Bosy-Westphal, A.; Szramek, A.; Scheufele, R.; Smoliner, C.; Pirlich, M. Cutoff percentiles of bioelectrical phase angle predict functionality, quality of life, and mortality in patients with cancer. Am. J. Clin. Nutr. 2010, 92, 612-619. [CrossRef]

64. Ørskov, M.; Iachina, M.; Guldberg, R.; Mogensen, O.; Nørgård, B.M. Predictors of mortality within 1 year after primary ovarian cancer surgery: A nationwide cohort study. BMJ Open 2016, 6, e010123. [CrossRef]

65. Park, H.S.; Lee, H.S.; Park, J.S.; Park, J.S.; Lee, D.K.; Lee, S.-J.; Yoon, D.S.; Lee, M.G.; Jeung, H.-C. Prognostic Scoring Index for Patients with Metastatic Pancreatic Adenocarcinoma. Cancer Res. Treat. 2016, 48, 1253-1263. [CrossRef] 
66. Penel, N.; Hollebecque, A.; Maynou, C.; Dewaele, J.; Jasserand, M.; Beuscart, R.; Vieillard, M.-H. Development of a score that predicts survival among patients with bone metastasis revealing solid tumor. Support. Care Cancer 2008, 16, 1089-1093. [CrossRef]

67. Penel, N.; Vanseymortier, M.; Bonneterre, M.-E.; Clisant, S.; Dansin, E.; Vendel, Y.; Beuscart, R.; Bonneterre, J. Prognostic factors among cancer patients with good performance status screened for phase I trials. Investig. New Drugs 2008, 26, 53-58. [CrossRef]

68. Pinato, D.J.; Karamanakos, G.; Ishizuka, M.; Smirne, C.; Pirisi, M.; Kubota, K.; Sharma, R. The Kings Score refines prognostic prediction in hepatocellular carcinoma: A novel application. Liver Int. 2015, 35, 2458-2465. [CrossRef] [PubMed]

69. Pointillart, V.; Vital, J.-M.; Salmi, R.; Diallo, A.; Quan, G.M. Survival prognostic factors and clinical outcomes in patients with spinal metastases. J. Cancer Res. Clin. Oncol. 2010, 137, 849-856. [CrossRef] [PubMed]

70. Roychowdhury, D.; Hayden, A.; Liepa, A. Health-Related Quality-of-Life Parameters as Independent Prognostic Factors in Advanced or Metastatic Bladder Cancer. J. Clin. Oncol. 2003, 21, 673-678. [CrossRef]

71. Rydzek, J.; Gąsior, Z.T.; Dąbek, J.; Wojnar, J.; Skrzypek, M. Assessment of risk factors for mortality in patients with cardiovascular disease and a history of treatment for malignancy. Kardiol. Polska 2015, 73, 730-739. [CrossRef]

72. Schoenfeld, A.J.; Ferrone, M.L.; Passias, P.G.; Blucher, J.A.; Barton, L.B.; Shin, J.; Harris, M.B.; Schwab, J.H. Laboratory markers as useful prognostic measures for survival in patients with spinal metastases. Spine J. 2020, 20, 5-13. [CrossRef]

73. Scott, H.R.; McMillan, D.C.; Forrest, L.M.; Brown, D.J.F.; McArdle, C.S.; Milroy, R. The systemic inflammatory response, weight loss, performance status and survival in patients with inoperable non-small cell lung cancer. Br. J. Cancer 2002, 87, 264-267. [CrossRef] [PubMed]

74. Seow, H.; Barbera, L.; Dudgeon, D.; Howell, D.; Husain, A.; Atzema, C.; Sussman, J.; Liu, Y.; Earle, C.; Sutradhar, R. The Association of the Palliative Performance Scale and Hazard of Death in an Ambulatory Cancer Population. J. Palliat. Med. 2013, 16, 156-162. [CrossRef]

75. Shen, H.; Agarwal, D.; Qi, R.; Chalasani, N.; Liangpunsakul, S.; Lumeng, L.; Yoo, H.; Kwo, P. Predictors of outcome in patients with unresectable hepatocellular carcinoma receiving transcatheter arterial chemoembolization. Aliment. Pharmacol. Ther. 2007, 26, 393-400. [CrossRef]

76. Soubeyran, P.; Fonck, M.; Blanc-Bisson, C.; Blanc, J.-F.; Ceccaldi, J.; Mertens, C.; Imbert, Y.; Cany, L.; Vogt, L.; Dauba, J.; et al. Predictors of Early Death Risk in Older Patients Treated with First-Line Chemotherapy for Cancer. J. Clin. Oncol. 2012, 30, 1829-1834. [CrossRef] [PubMed]

77. Sutradhar, R.; Atzema, C.; Seow, H.; Earle, C.; Porter, J.; Barbera, L. Repeated Assessments of Symptom Severity Improve Predictions for Risk of Death Among Patients with Cancer. J. Pain Symptom Manag. 2014, 48, 1041-1049. [CrossRef] [PubMed]

78. Suzuki, H.; Ito, M.; Takemura, K.; Nakanishi, Y.; Kataoka, M.; Sakamoto, K.; Tobisu, K.-I.; Koga, F. Prognostic significance of the controlling nutritional status (CONUT) score in advanced urothelial carcinoma patients. Urol. Oncol. Semin. Orig. Investig. 2020, 38, 76.e11-76.e17. [CrossRef]

79. Tsai, H.-J.; Hsieh, M.-Y.; Tsai, Y.-C.; Liu, Z.-Y.; Hsieh, H.-Y.; Lee, C.-M.; Chien, C.-H.; Chiu, Y.-W.; Chuang, H.-Y.; Huang, C.-T. Liver function tests may be useful tools for advanced cancer patient care: A preliminary single-center result. Kaohsiung J. Med. Sci. 2014, 30, 146-152. [CrossRef]

80. Tripodoro, V.A.; Llanos, V.; de Lellis, S.; Salazar Güemes, C.; de Simone, G.G.; Gómez-Batiste, X. Prognostic factors in cancer patients with palliative needs identified by the necpal CCOMS-ICO@ tool. Medicina 2019, 79, 95-103. [PubMed]

81. Ueno, H.; Okada, S.; Okusaka, T.; Ikeda, M. Prognostic Factors in Patients with Metastatic Pancreatic Adenocarcinoma Receiving Systemic Chemotherapy. Oncology 2000, 59, 296-301. [CrossRef]

82. Van der Linden, Y.M.; Dijkstra, S.P.; Vonk, E.J.; Marijnen, C.A.; Leer, J.W.; Dutch Bone Metastasis Study Group. Prediction of survival in patients with metastases in the spinal column: Results based on a randomized trial of radiotherapy. Cancer 2005, 103, 320-328. [CrossRef]

83. Viganó, A.; Bruera, E.; Jhangri, G.S.; Newman, S.C.; Fields, A.L.; Suarez-Almazor, M.E. Clinical Survival Predictors in Patients with Advanced Cancer. Arch. Intern. Med. 2000, 160, 861-868. [CrossRef]

84. Villà, S.; Weber, D.C.; Moretones, C.; Mañes, A.; Combescure, C.; Jové, J.; Puyalto, P.; Cuadras, P.; Bruna, J.; Verger, E.; et al. Validation of the new graded prognostic assessment scale for brain metastases: A multicenter prospective study. Radiat. Oncol. 2011, 6, 23. [CrossRef]

85. Wei, J.; Tang, D.; Nie, Y.; Chen, J.; Peng, L. Clinical characteristics and prognosis of nonsurgically treated patients with pneumonictype adenocarcinoma. Medicine 2019, 98, e15420. [CrossRef]

86. Yamashita, T.; Siemionow, K.; Mroz, T.; Podichetty, V.; Lieberman, I.H. P89. A Prospective Analysis of Prognostic Factors in the Patients with Spinal Metastases Use of the Revised Tokuhashi Score. Spine J. 2008, 8, 143S-144S. [CrossRef]

87. Stone, P.; Lund, S. Predicting prognosis in patients with advanced cancer. Ann. Oncol. 2007, 18, 971-976. [CrossRef] [PubMed]

88. Blanchon, F.; Grivaux, M.; Asselain, B.; Lebas, F.-X.; Orlando, J.-P.; Piquet, J.; Zureik, M. 4-year mortality in patients with non-small-cell lung cancer: Development and validation of a prognostic index. Lancet Oncol. 2006, 7, 829-836. [CrossRef]

89. Bollen, L.; van der Linden, Y.M.; Pondaag, W.; Fiocco, M.; Pattynama, B.P.M.; Marijnen, C.; Nelissen, R.; Peul, W.C.; Dijkstra, P.S. Prognostic factors associated with survival in patients with symptomatic spinal bone metastases: A retrospective cohort study of 1043 patients. Neuro-Oncology 2014, 16, 991-998. [CrossRef] [PubMed]

90. Westhoff, P.G.; de Graeff, A.; Monninkhof, E.M.; Bollen, L.; Dijkstra, S.P.; van der Steen-Banasik, E.M.; van Vulpen, M.; Leer, J.W.H.; Marijnen, C.A.; van der Linden, Y.M. An Easy Tool to Predict Survival in Patients Receiving Radiation Therapy for Painful Bone Metastases. Int. J. Radiat. Oncol. 2014, 90, 739-747. [CrossRef] [PubMed] 
91. Hui, D.; Paiva, C.E.; Del Fabbro, E.G.; Steer, C.; Naberhuis, J.; van de Wetering, M.; Fernandez-Ortega, P.; Morita, T.; Suh, S.Y.; Bruera, E.; et al. Prognostication in advanced cancer: Update and directions for future research. Support. Care Cancer 2019, 27, 1973-1984. [CrossRef]

92. Maas, E.A.T.; Murray, S.A.; Engels, Y.; Campbell, C. What tools are available to identify patients with palliative care needs in primary care: A systematic literature review and survey of European practice. BMJ Support. Palliat. Care 2013, 3, 444-451. [CrossRef]

93. Romo, R.; Lynn, J. The utility and value of the "surprise question" for patients with serious illness. Can. Med. Assoc. J. 2017, 189, E1072-E1073. [CrossRef]

94. Walsh, R.I.; Mitchell, G.; Francis, L.; van Driel, M.L. What Diagnostic Tools Exist for the Early Identification of Palliative Care Patients in General Practice? A systematic Review. J. Palliat. Care 2015, 31, 118-123. [CrossRef] 